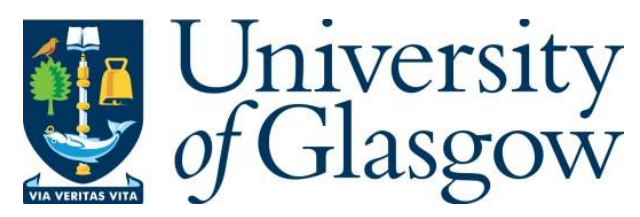

Munyikwa, K., Kinnaird, T. C. and Sanderson, D. C.W. (2021) The potential of portable luminescence readers in geomorphological investigations: a review. Earth Surface Processes and Landforms, 46(1), pp. 131-150.

There may be differences between this version and the published version. You are advised to consult the publisher's version if you wish to cite from it.

http://eprints.gla.ac.uk/249952/

Deposited on: 20 August 2021

Enlighten - Research publications by members of the University of Glasgow http://eprints.gla.ac.uk 


\section{The potential of portable luminescence readers in geomorphological}

\section{2 investigations: a review}

3

4 Ken Munyikwa ${ }^{1}$, Tim C. Kinnaird ${ }^{2}$, and David C.W. Sanderson ${ }^{3}$

5

$6 \quad{ }^{1}$ Centre for Science, Athabasca University, Athabasca, Alberta, Canada

$7{ }^{2}$ School of Earth and Environmental Sciences, University of St Andrews, St Andrews, 8 Scotland, U.K.

$9{ }^{3}$ Scottish Universities Environmental Research Centre, East Kilbride, Glasgow, Scotland, 10 U.K.

\section{Correspondence}

12 Ken Munyikwa, Centre for Science, Athabasca University, 1 University Drive, Athabasca, 13 AB T9S 3A3, Canada,

14 kenm@athabascau.ca

\section{ABSTRACT}

17 The development of functional portable optically stimulated luminescence (OSL) readers 18 over the last decade has provided practitioners with the capability to acquire 19 luminescence signals from geological materials relatively rapidly, which allows for 20 expedient preliminary chronostratigraphic insight when working with complex depositional 21 systems of late Quaternary age. Typically, when using the portable OSL reader, infrared 22 (IR) or blue post-IR OSL signals are acquired from bulk unprocessed materials, in 
24 quartz or feldspar mineral separates, or on select silt-sized polymineralic portions. To 25 demonstrate the utility of portable OSL measurements, this paper outlines the basic features of portable OSL readers and their constraints. Afterwards, case studies in which

27 the instrument has been used to elucidate cryptostratigraphic variations in sedimentary sequences for geomorphological applications are reviewed. The studies can generally be grouped into three main categories. The first includes studies where the variation of portable OSL reader luminescence signal intensities with depth are plotted to generate profiles that contextualise sediment stratigraphy. In the second group, portable OSL reader luminescence signal intensities are used to interpret sediment processes that shed light on depositional histories. In the last category, luminescence signals from the portable OSL reader are calibrated to approximate numerical burial ages of depositional units. The paper concludes with a discussion of possible future directions.

Keywords geomorphology, dating, landscape evolution, optically stimulated luminescence, portable OSL reader, chronology

\section{INTRODUCTION}

42 Luminescence dating is an effective dating approach in the quantitative study of late Quaternary clastic depositional systems (e.g. Aitken, 1998; Wintle, 2008; Rhodes, 2011). 44 Absolute ages acquired using the method help assign temporal frameworks to geomorphic events and environmental processes. The dating method is based on the ability of some minerals, particularly quartz and feldspar, to cumulatively store energy in

47 the form of trapped charges produced by ionizing environmental radiation (Aitken, 1998). 
48 Exposure of the mineral grains to sunlight causes the energy to be lost, a process also referred to as bleaching. Hence, the accumulation of the energy only begins once the sediment grains are buried. Collecting the mineral grains from the field and stimulating

51 them in a laboratory allows the accumulated energy (paleodose) to be assessed.

52 Typically for sediments, stimulation is performed using a light source to yield optically 53 stimulated luminescence (OSL). Determining the rate at which the energy accumulated 54 (dose rate) allows the burial age of the sediment to be calculated by dividing the

55

56

57

58

paleodose by the dose rate. In natural environments, the burial of sediments is normally driven by geomorphic processes. Thus, luminescence dating ascertains time that has passed following the occurrence of a particular geomorphic episode. Accordingly, sediments that can be dated using luminescence methods essentially comprise clastic deposits that were appropriately bleached by sunlight before burial.

Full-fledged luminescence dating using standard protocols that employ laboratory-based luminescence readers is a time- and resource-intensive procedure. The method requires meticulous extraction of pure mineral separates (usually quartz or potassium feldspar) when using the coarse-grain method, or the fine-grain quartz approach. When dating siltsized particles using feldspar signals, polymineralic grains within a specific grain size range are extracted instead. Furthermore, unique growth curves that reflect the relationship between the luminescence signal and the energy dose have to be constructed for each sample following the administration of artificial irradiation in order to calibrate the natural dose received by a sample. Apart from the requirement that the sample should have been adequately zeroed prior to being buried, sediment samples 
71 have to fulfill a number of other pre-requisites before they can yield a reliable age. These

72 include that electron traps which store the charge within the dosimeter lattices should not

73 be exhausted or saturated prior to the end of the period being dated (e.g. Aitken, 1998),

74 that initial zeroing has been complete, and that the sample has not been subject to post75 depositional disturbance.

77 Because of the time- and resource-intensive character of conventional luminescence dating using standard instrumentation, many luminescence dating studies typically 79 feature a relatively smaller number of ages than would be desirable under ideal circumstances. When working with complex geomorphological settings (e.g. Kocureck 81 and Ewing, 2005), the limited spatial density of the ages is often insufficient to render a 82 comprehensive understanding of the stratigraphic evolution of the landscape in question (e.g. Telfer, 2011; Munyikwa et al., 2012). The effect is exacerbated when collecting samples from depositional sequences that have no previous age information as the absence of an overarching chronological framework does not permit samples to be extracted from the most appropriate deposition units. Overall, these aspects highlight the necessity for acquiring appropriate chronological insight into the stratigraphic setting of any site under investigation as well as the potential benefits of conducting rigorous screening of samples that are ultimately selected for dating using standard luminescence dating protocols. Such precautions help prevent the expenditure of resources and time

91 on samples that are not suitable for dating. Methods that have recently been used to 92 provide such insight include the use of ground penetrating radar to examine internal dune 93 structure (e.g. Bristow, et al., 2007). 
95 Over the last decade, the development of functional portable OSL readers (e.g. Sanderson and Murphy, 2010; Kook et al. 2011) has provided researchers with a practical option to obtain luminescence signals from dosimeters relatively rapidly, compared to when using standard lab-based luminescence readers. The portable OSL reader, which can be acquired at a fraction of the cost of a standard OSL reader, enables researchers to attain a quick approximation of the luminescence energy stored in a sample for three main reasons. First, since the device is lightweight and portable, it can be easily transported to the field site: sample measurement can be conducted contemporaneously with sample collection. Second, the simplicity of the measurements, which in most cases can be performed without sample preheating, allows for quick results. Third and most importantly, the portable OSL reader can typically be used on bulk unprocessed sediment and this truncates the analytical process significantly compared to regular OSL dating. Overall, the combined effect of these aspects is that larger numbers of samples can be analyzed more rapidly and at much lower cost using the portable OSL reader. For any given study, the ability to analyze more samples introduces a greater spatial resolution of chronostratigraphic data which, in turn, affords practitioners prompt and improved contextual insight into geomorphic processes pertaining to landscape evolution.

Notably, however, there are a number of intrinsic drawbacks associated with the use of bulk, unseparated samples and portable luminescence readers compared to conversional lab-bound systems. These include: 
- First, unlike the case with regular OSL readers, luminescence signals obtained with the portable instrument cannot readily be used to generate absolute ages using standard protocols. This is not least because signals from the unprocessed bulk samples often comprise mixed signals (e.g. mixed quartz and feldspar signals obtained under blue-OSL stimulation) because no mineralogical separations are performed prior to analysis. While pulsed methods have been used in some studies to partially separate quartz and feldspar signals (e.g. Kook et al., 2011) to date beyond approximations used in reconnaissance studies (e.g. Munyikwa and Brown, 2014; Stone et al., 2019) the combination of bulk screening and portable readers has not generally been used to produce full-fledged OSL ages. Bulk samples often contain a wide spectrum of grain sizes that may range from clay and silt fractions to coarse sand. In conventional OSL dating, specific grain size ranges are usually extracted and analyzed separately since they generally exhibit different luminescence characteristics, and their microdosimetry depends significantly on grain size and internal radioactivity levels. As outlined below, the lack of pre-heating capability or of irradiation sources in some portable systems while increasing portability precludes the field implementation of full-fledged OSL dating procedures.

- Second, when comparing raw portable OSL reader luminescence signals obtained from different units within a depositional sequence, direct comparison of signal intensities as indicators of relative chronology can only be made if the co-factor variables that influence signal intensity (e.g. mineralogy, dose rate, grain size, 
aliquot size, degree of bleaching before burial, luminescence sensitivity, etc.) are constant between units. This is not always the case and therefore interpretation of co-factors and their potential variations is an important part of both initial field interpretation, and of subsequent stages of evaluation.

- Third, apart from applications that aim to approximate equivalent dose in samples, signals obtained using the portable OSL reader are often not routinely normalized for the range of variables that influence the signal intensity. This makes it difficult to compare raw signals from disparate sites, with most comparisons being confined to samples collected from proximal locations where dose rate, mineralogy, granulometry, etc. are thought to be consistent.

Nonetheless, despite these drawbacks, if the use of portable luminescence readers is considered complementary to the application of conventional OSL readers rather than a replacement, some of the potential disadvantages of the portable systems become inconsequential.

To highlight the utility of the portable OSL reader in geomorphological applications and to examine the current state of the science, this paper reviews studies that have been conducted over the last decade, identifying areas the investigations have focused on. In order to familiarize the reader with the basic layout of the portable OSL readers, design aspects of instruments developed over the last decade and their operational features are discussed. Studies in which the portable OSL reader has been used are then explored and these can generally be grouped into three main categories. The first comprises 
163 studies in which portable OSL readers have been used to construct vertical luminescence 164 profiles that show the variation of luminescence signals intensities with depth in 165 depositional sequences. In settings where the main determinant of signal intensity is 166 sediment age, the luminescence profiles serve as proxies for the chronostratigraphy (e.g. 167 Sanderson and Murphy, 2010; Muñoz-Salinas et al., 2011; Kinnaird, et al., 2015). Hence, 168 they can provide relative ages of depositional units and would be indispensable when 169 formulating a sampling strategy for full-fledged dating. Additionally, portable OSL reader 170 luminescence signals could provide insight into the geomorphic processes involved. In 171 the second category, luminescence measurements obtained using a portable OSL reader 172 are used to interpret sediment processes by examining bleaching characteristics of 173 sediments from various depositional environments. The data are used to interpret 174 sediment depositional pathways in order to gain a better understanding of landscape 175 evolution. Sediment properties such as luminescence sensitivity can also be examined 176 allowing them to be used as tracers for provenance studies (e.g. Gray et al., 2019; 177 Lichtenberger et al., 2019). In the third category, luminescence signals from the portable 178 OSL reader are calibrated in order to use them to approximate numerical burial ages of 179 depositional units (e.g. Munyikwa and Brown, 2014; Stone et al., 2015; Stone et al., 2019). 180 For each of the three categories, the environmental context in which the portable OSL 181 reader was used is examined and the methodological aspects explored. The paper 182 concludes with a brief look at future possible developments in methodological approaches 183 as well as instrumental design. Overall, the scope of the paper and the case studies 184 discussed are limited to geoscience applications completed so far. Thus, we do not 185 examine theoretical and developmental aspects that would be more appropriate in a 
186 separate dedicated review. This is tacit recognition that significant other research is in 187 progress, most of which does not yet appear in the literature.

\section{BASIC LAYOUT OF THE PORTABLE LUMINESCENCE READER}

The potential benefits of being able to conduct a quick assessment of the luminescence properties of depositional sequences in the field has long been appreciated in geology and geomorphology. Concepts of portable luminescence readers that reached the development stage over the last three decades include instruments reported by Poolton et al. (1994), Takeuchi et al. (2008), Sanderson and Murphy (2010), and Kook et al. (2011). Each of these instruments is discussed briefly below. A portable OSL reader developed by Smetana et al. (2008) is largely intended for assessing exposure to UV radiation in work settings. Though the instrument could theoretically be adapted for assessing ionising radiation for geological applications, it will not be examined in this paper. In Table 1, potential advantages and drawbacks of each design are explored.

The instrument developed by Poolton et al. (1994) weighed about $5 \mathrm{~kg}$ and was mainly designed for analyzing feldspar. However, with extensions, quartz could be examined too.

207 The instrument featured a $30 \mathrm{~mm}$ photomultiplier (PM) tube (EMI bialkali photocathode, 208 type 9924B) for photon detection after passing through a Schott BD39 filter. Sample 
209 stimulation was provided by IR light emitting diodes (LEDs) supplying about $30 \mathrm{mM} / \mathrm{cm}^{2}$ 210 of power $(880 \mathrm{~nm})$ that targeted feldspar in continuous wave (CW) mode. Samples were 211 held in a cartridge that could hold up to 12 samples in the form of pellets or sand grains. 212 Bleaching capability was rendered by a blue Osram (DS/E 9-71) fluorescence lamp (400$213550 \mathrm{~nm}$ ) while $\mathrm{Hg}$ discharge UV lamps emitting around $50 \mu \mathrm{W} / \mathrm{cm}^{2}$ at $254 \mathrm{~nm}$ (Osram 214 HN10/UOFR or HNS10/UOZ) were used as an excitation source for signal normalisation. 215 The instrument operated on a $12 \mathrm{~V}$ power source that could be supplied by the mains grid 216 or by a portable source such as an automotive battery. A laptop computer provided a user 217 interface. By adding a tungsten-halogen stimulation source to the system, the instrument 218 could be expanded to also measure quartz OSL.

223 Similar to the design by Poolton et al. (1994), the reader developed by Takeuchi et al. 224 (2008) has a multiple sample holder with 13 positions. Weighing about $15 \mathrm{~kg}$, the unit can 225 perform both thermal (red thermoluminescence- RTL) and optical (OSL) stimulation. The 226 optical stimulation is achieved by 16 blue $(470 \mathrm{~nm})$ LEDs, targeting quartz as well as 16 227 IR $(890 \mathrm{~nm})$ LEDs intended for feldspars. When conducting stimulation with the blue 228 LEDs, SC42 (FUJI Photo Film) filters are used to screen the source signal before it 229 reaches the sample. Four ceramic heaters enclosed in a brass casing provide the heating 230 and can attain up to $600^{\circ} \mathrm{C}$ with electrical power under $128 \mathrm{~W}$. A meta-packaged PM 231 tube (Hamamatsu Photonics H7421-40) performs photon detection and OSL signals need 
232 pass through a DUG11 filter (Schott) with a detection range of $300-400 \mathrm{~nm}$ before

233 measurement. Artificial irradiation of samples is rendered using a miniature X-Ray 234 generator (Oxford Eclipse-II-Reflection) that requires electrical power of around $3 \mathrm{~W}$. The 235 device can perform both CW and pulsed OSL measurements. Total power required to run 236 the instrument without heating is about $50 \mathrm{~W}$ and when the laptop and heating are 237 included, about $128 \mathrm{~W}$ is needed. In pulsed mode, pulse widths can range from $2-10 \mu \mathrm{s}$ 238 while the interval between the pulses ranges between 200-1600 $\mu$ s. Pulsed-OSL signals 239 are recorded between pulses.

The portable OSL reader system used in all the studies discussed in this paper is the 245 instrument developed by the Scottish Universities Environmental Research Centre (SUERC) and was described by Sanderson and Murphy (2010). We have not been able 247 to identify any published studies that have employed the other three portable OSL readers 248 and none of them appear to have progressed beyond the prototype instrument. We 249 suspect the reason users have predominantly preferred the SUERC portable OSL reader 250 so far is because of its ruggedness and operational simplicity. The basic layout of first251 and second-generation designs of the SUERC portable OSL reader (up to ca. 2015) 252 comprised three components: a detector-head mounted on a sample drawer, a control 253 box with the operating switchgear, and a laptop computer to provide a user interface and 254 a data logging system. The drawer holds samples that are introduced in 5-centimeter 
255 diameter petri dishes or planchettes and the luminescence signal is obtained following 256 stimulation using the appropriate source. The stimulating sources are housed just below 257 the detector-head and normally comprise IR LEDs centred around $880 \mathrm{~nm}$, as well as 258 blue LEDs centred around $470 \mathrm{~nm}$. Ports for the IR diodes are equipped with RG780 long 259 pass filters while the blue LED ports are fitted with GG420 long pass filters. Following 260 stimulation, the luminescence signal passes through UG11 filters and is detected by an 261 ETL photon detector module. Fixed filters are used in the systems for ruggedness. But 262 stimulation cones with different wavelength sources and detections bands have also been 263 produced, allowing for different configurations. Sample stimulation can be in CW or 264 pulsed mode. Pulse-on and pulse-off period can be set between 1 and $99 \mu$ s (Sanderson 265 and Murphy, 2010). In addition to the switchgear, the control box also holds $4 \mathrm{NiMH} 1.25$ $266 \mathrm{~V}$ batteries that can be used to provide power to the system when not connected to the 267 mains grid. Overall, the instrument weighs under $5 \mathrm{~kg}$. The third-generation SUERC 268 portable OSL reader, which has been in operation since 2015 , combines the detector 269 head and sample tray as a single module (Figure 1). Other components including the 270 electronics and operating software remain relatively similar to the second-generation 271 design. 
279 The fourth and final luminescence reader examined in this paper is the portable 280 instrument designed through collaboration between the Nordic Laboratory for 281 Luminescence Dating and the Korea Basic Science Institute (KBSI). The device weighs 282 about $8 \mathrm{~kg}$ and runs on a DC source (9-45V) or, alternatively, on grid power (AC). What 283 differentiates this instrument from other portable OSL readers is a specially designed 284 sampler that can be inserted into a depositional unit in its natural setting. This avoids the 285 need for light-free conditions to enable the transfer of samples into a holder during 286 measurement. The sampler can hold three samples and heat materials at $3^{\circ} \mathrm{C} \mathrm{s}^{-1}$ up to $287250^{\circ} \mathrm{C}$ using a heating coil (ThermoCoax) paired with a thermo sensor (RTD, Pt100). 288 Other components of the system comprise the main body of the instrument which serves 289 as the measurement table. Above the measurement table is a measurement head that 290 houses the PM tube, LEDs for stimulation, as well as the X-ray source for artificial 291 irradiation. For stimulating samples, the instrument only uses a blue OSL source 292 comprising 24 W LEDs centred around $470 \mathrm{~nm}$ for analyzing quartz. A long pass GG420 293 Schott filter is placed in front of the LEDs. Luminescence signals are detected by a 30 $294 \mathrm{~mm}$ bialkali PM tube (9125B, ET Enterprises Limited) after passing through a U340 filter 295 (Hoya). As with the SUERC reader, Kook et al.'s (2011) instrument can also operate in 296 both CW and pulsed mode. When in pulsed mode, pulse-on and pulse-off period can be 297 set anywhere between 1 and 65,535 $\mu$ s. Sample heating capability allows TL analysis to 298 be made. Apart from measuring regular luminescence, the device can also measure 299 radioluminescence, which is the signal obtained while a sample is irradiated by an ionizing 300 source. 


\subsection{Portable OSL reader measurement modes}

Both regular and portable OSL readers are capable of performing measurements on processed as well as on unprocessed samples. Bulk samples are usually analyzed when prompt data are required for reconnaissance or screening purposes. Portable OSL readers optimize the ability to acquire speedy results from bulk samples because they

312 can be taken into the field where near real-time data can be incorporated into the sampling 313 strategy. Sample mounting is also far simpler in some portable OSL readers than in the 314 standard lab-bound varieties. In many depositional settings, bulk materials would include 315 both quartz and feldspar. Hence, the measurement strategy that is usually adopted aims 316 to target grains of quartz or feldspar in separate measurement steps performed at room

317 temperature. Since IR stimulation has a negligible effect on the fast component of quartz 318 below $125^{\circ} \mathrm{C}$ (Spooner and Questiaux, 1989; Short and Huntley, 1992; Bailey, 1998; 319 Thomsen et al., 2008), measurement on polymineralic aliquots is typically conducted by 320 first stimulating using an IR source after which blue OSL stimulation may follow. Notably, 321 blue light causes luminescence in both feldspars and quartz. However, a significant 322 proportion of blue-sensitive traps in feldspar are also depleted by prolonged exposure to 323 IR stimulation (Duller and Bøtter Jensen, 1993; Clark and Sanderson, 1994; Galloway, 
324 1994; Jain and Singhvi, 2001). Hence, stimulating the sample with blue-OSL after IR

325 stimulation may enhance the quartz contribution from bulk samples. The OSL signal

326 obtained from this sequence of analysis is referred to as a post-IR blue OSL signal (e.g.

327 Roberts and Wintle, 2001; Wallinga et al., 2002). When using the SUERC reader in CW

328 mode, it is possible to use the sequence editor in the user software to vary both the dark

329 count and the times of exposure to IR or OSL sources. The dark count measurement

330 mode provides the machine background count rate in the absence of stimulation, and

331 defines the statistical detection limits of detection of weak luminescence signals. Dark

332 counts originating from the photomultiplier comprise both thermal and non-thermal

333 components (Carter et al, 2018). The measurement sequence can also be used to record

334 low level [phosphorescence emitted from samples when first introduced to the system, or

335 post-stimulation phosphorescence, which is emitted at low levels after the samples have

336 been measured. Dark count measurements can be alternated with stimulation

337 measurement modes. Stimulation times can vary from 0-999 s. Typical measurement

338 sequences involve a $10 \mathrm{~s}$ dark count followed by 60-80 s IRSL or OSL stimulation after

339 which another dark count is taken. Post-stimulation phosphorescence (PSP) can also be 340 monitored if needed.

342 Quartz and feldspar signal separation under blue OSL stimulation can also be performed 343 by pulsing the OSL signal. Luminescence of feldspars includes shorter lifetimes in the 344 nanosecond to several microsecond timescales than those associated with the main 345 quartz OSL emissions (e.g. Sanderson \& Clark 1994, Denby et al., 2006; Thomsen et al., 346 2008; Ankjaergaard et al., 2015). Hence, the suggestion that in pulsed mode, by 
347 performing the measurement of the sample signal only during the pulse-off period and 348 delaying the onset of the measurement (e.g. by $2-5 \mu \mathrm{s}$ ), the faster feldspar signal 349 components can be suppressed while allowing quartz dominant signals to be measured. 350 Kook et al. (2011) primarily used this approach to concentrate quartz signals from 351 samples that contain both quartz and feldspar grains.

353 Typical IRSL and post-IR blue OSL shine-down curves obtained using a SUERC reader 354 in both CW and pulsed modes are shown in Figure 2. The slow depletion of the signals 355 are the result of the relatively low power from the stimulation sources, coupled with the 356 deliberately large sample areas, and the use of thick samples (e.g. Stone et al., 2015). 357 Additionally, feldspar contribution to the post-IR blue OSL signal could also be influencing 358 the depletion rate (e.g. Duller, 2003). As detailed above, when in pulsed mode, the 359 SUERC portable OSL reader cannot gate the signal measurement to pulse-off periods 360 only such that feldspar emissions, if present, are not excluded. Pulsed stimulation using 361 a SUERC portable OSL reader was reported by Muñoz-Salinas et al. (2011). In this case 362 pulse-on window was synchronized with measurements on a 15 microsecond gate, thus 363 autosubtracting dark count signals and any long-term luminescence recombination. 
369 IRSL or OSL measurements from the portable OSL reader are often presented as 370 integrated signal intensities over the period of measurement. It is also possible to 371 calculate signal depletion ratios, IRSL/OSL ratios, post-stimulation IRSL and OSL PSP 372 (Sanderson and Murphy, 2010; Kinnaird et al., 2015; Kinnaird et al., 2017). The signal 373 depletion index is calculated as the ratio of the luminescence intensity in the first half of 374 the stimulation period divided by the intensity in the second half. Factors that can 375 influence the depletion rate of bulk samples include the mineralogy of the sample, grain 376 size distribution, color of the grains (or coatings) as well as the extent to which a sample 377 contains mixtures of grains that were well beached and those that were partially bleached 378 prior to the last burial event (Sanderson and Murphy, 2010). Depletion ratios of sediments 379 that were well bleached prior to burial would be higher than for sediments that were not 380 completely bleached since inherited signals deplete less rapidly. Overall, the use of 381 depletion ratios allows a determination to be made if variations in signal intensity down a 382 sequence are influenced by factors other than burial age or dose rate. For instance, 383 Sanderson et al. (2010) examined depletion ratios for a stratigraphic sequence 384 comprising Neolithic ditch fills at Cava Petrilli, Italy. Results showed the ratios were 385 relatively constant down the section, suggesting that bulk properties such as sediment 386 color and grain size did not lead to significant variations in luminescence sensitivity 387 between the different depositional units. In another study, examining agricultural terraces 388 from Catalonia in eastern Spain, Kinnaird et al. (2017) noted that depletion ratios provided 389 an indication that the units were better bleached at deposition, and used this to identify 390 anthropogenic and natural fills. In essence, the depletion ratio allows main determinants 391 of the variations in luminescence intensity to be identified. 
Similarly, if IRSL measurements are targeted at feldspar, and OSL at quartz, comparisons of IRSL/OSL ratios between different samples could be seen as a reflection of variations in relative concentrations of feldspar to quartz within the samples. Stratigraphic sections that feature homogenous IRSL/OSL ratios (e.g. Munyikwa et al., 2012) would suggest 397 that proportions of feldspar relative to quartz are constant down the sequence, and that variations in luminescence intensity arise from other influences such as burial age or dose rate. Conversely, sections where IRSL/OSL values fluctuate point to variations in the relative concentrations of feldspar to quartz. Such variations have been attributed to 401 differences in the degree of weathering (e.g. Sanderson and Murphy, 2010) since 402 feldspars disintegrate more readily than quartz when exposed to the elements.

\section{CONTEXTUALIZING SEDIMENT STRATIGRAPHY BY LUMINESCENCE}

\section{PROFILING USING THE PORTABLE OSL READER}

407

\subsection{Luminescence profiling using conventional OSL readers}

409

Before discussing luminescence profiling using portable OSL readers, it is pertinent to

411 examine profiling that can be performed using standard lab-bound OSL readers. When

412 initially proposed, the primary aim of profiling using standard OSL readers was to acquire

413 preliminary insight into depositional contexts of stratigraphic sites and to assess the 414 suitability of samples for conventional OSL dating (e.g. Sanderson et al., 2001; 2003; 
415 Burbidge et al., 2007). A key aspect of this approach is the use of bulk or partially 416 processed samples as well as abbreviated procedures for determining $D_{e}$ in order to 417 expedite the evaluation. For instance, working on Paleolithic archeological sites in Russia, 418 Burbidge et al. (2007) encountered a range of stratigraphic sequences some of which did 419 not appear to be suitable for dating using OSL. Hence, an initial evaluation of the sites 420 was performed to identify samples worth dating using full-fledged OSL protocols. Aspects 421 the preliminary assessment aimed to address included the extent to which sediment 422 signals had been reset prior to the last burial episode and the identification of discrete 423 depositional phases contained in the sections. Ages that had been obtained from the 424 sequences in previous studies using ${ }^{40} \mathrm{Ar} /{ }^{39} \mathrm{Ar}$ dating, ${ }^{14} \mathrm{C}, \quad \delta^{18} \mathrm{O}$ stratigraphy, 425 magnetostratigraphy, as well as conventional OSL provided independent age controls for 426 the sections. As part of the profiling, samples collected from the stratigraphic sites were 427 divided into three separate subpopulations: polymineral silt $(4-12 \mu \mathrm{m})$, polymineral sand $428 \quad(90-250 \mu \mathrm{m})$, as well as quartz-enriched sand $(90-250 \mu \mathrm{m})$. In this way, the influence of 429 grain size and mineralogy on luminescence signals was examined. Each of the three 430 subpopulations was measured using IRSL, post-IR OSL and TL on a conventional OSL 431 reader, and equivalent doses $\left(D_{e}\right)$ calculated. The $D_{e}$ determinations employed an 432 abbreviated regenerative dose method that only used two aliquots. Values that were 433 obtained were then plotted to show the variation of $D_{e}$ with depth for each section. Results 434 indicated that data obtained using coarse-gained polymineral sand were relatively 435 consistent with those obtained using quartz-enriched sand, suggesting that rapid 436 measurements using partially processed samples and abbreviated protocols could 437 produce valuable preliminary information. Furthermore, the approach showed that 
438 combining rapidly acquired profiling results from a given site with a few ages obtained 439 using standard OSL protocols could yield a detailed chronostratigraphic framework, 440 ultimately conserving time, effort and resources.

442 Other studies that have examined ways of conducting preliminary studies using standard 443 OSL readers include work by Hamel and Huntley (2003), who used IRSL to estimate 444 equivalent dose in unprocessed sand and noted that measurements made on the 445 samples returned $D_{e}$ estimates that were reliable approximations of values obtained using 446 standard OSL protocols. Possible reasons for variations of results between the two 447 approaches that were cited included the broader grain size range in the raw samples and 448 the possible presence of other minerals that responded to IRSL, unlike in the processed 449 samples where only K-feldspar was present.

451 Beyond investigating depositional sites using luminescence profiling for the presence of 452 samples suitable for dating (e.g. Sanderson et al., 2001, 2003; Burbidge, 2007), signals 453 obtained from quartz in bulk samples using standard OSL readers have also been used 454 to determine OSL range-finder ages that give preliminary age estimates (e.g. Roberts et 455 al., 2009; Durcan et al., 2010). Sand from a dune from Namib desert as well as from a 456 coastal dune from the UK were analyzed by Roberts et al. (2009), by comparing $D_{e}$ values 457 obtained from raw samples to values yielded by pure quartz from the same sample. 458 Results showed that raw samples returned $D_{e}$ values within $65-70 \%$ of the $D_{e}$ obtained 459 from pure quartz. When the bulk sample was first exposed to IR stimulation for $500 \mathrm{~s}$ 460 before post-IR blue OSL stimulation, the $D_{e}$ values calculated were within $82-90 \%$ of the 
461 value obtained from pure quartz separates. Hence, this suggested that reliable range-

462 finder ages could be estimated on quartz in raw samples, especially using post-IR OSL

463 stimulation. However, the same result was not replicated in another study. Working with

464 samples from eastern Pakistan, Durcan et al. (2010) noted that the initial IR stimulation

465 could not adequately deplete the feldspar signal such that post-IR blue OSL signals

466 continued to be dominated by feldspar emissions. As a result, further chemical treatment

467 of the sample was required to eliminate feldspar before measurement. In other studies,

468 pulsed OSL signals using standard OSL readers (e.g. Thomsen et al., 2008) have been

469 used to measure quartz signals in samples with polymineral grains in combination with

470 minimal sample processing. Results showed close agreement between samples

471 prepared using conventional protocols and those that had simply been washed and

472 sieved.

473

474 Overall, what all these methods highlight is the inherent value of preliminary screening

475 methods that can be used to identify samples that are worth expending resources and

476 time on. They also show that useful information about the dose stored in a sample can

477 be ascertained by performing measurements on bulk samples with minimal or no 478 preprocessing. Ultimately, the efforts allow the extraction of maximum benefits from a 479 given study.

480

481

$482 \quad 3.2$ Luminescence profiling using portable OSL readers 
484 The portable OSL reader can be used to collect data for luminescence profiling as with 485 the conventional OSL reader. The main advantage of the portable OSL reader in this 486 case, as highlighted earlier, is that it can be taken to the field, allowing for rapid decisions 487 to be made, especially if the information is to be factored into a larger sampling program 488 for conventional OSL dating. However, the lack of an internal irradiation source in some 489 portable readers means that such devices cannot be used to normalize luminescence 490 signals for grain size variation or sample size using a test dose administered internally by 491 the reader. Despite that constraint, as will be shown in case studies examined below, 492 profiling using the portable OSL reader can provide invaluable preliminary insight into 493 cryptostratigraphic features of late Quaternary depositional systems (Sanderson and 494 Murphy, 2010). In most cases performed to date, profiles derived using portable reader 495 data are obtained by constructing vertical sections that show the variation of 496 luminescence signal intensities with depth or, in essence, luminescence stratigraphies 497 (e.g. Sanderson and Murphy, 2010). The signals plotted in a luminescence profile could 498 be either IRSL, or OSL net signal intensities that are recorded during measurement. 499 Depletion ratios and IRSL/OSL ratios can also be presented in profile form. As detailed 500 above, luminescence intensities recorded from the bulk samples depend on variables that 501 include a) the local dose rate, b) inherited dose at time of burial, c) luminescence 502 sensitivity of the mineral grains, d) time that has lapsed since burial age of the sediment, 503 and e) mineral composition. In a depositional sequence where all these variables are 504 constant, apart from the duration of burial, the profile would essentially be representative 505 of the chronostratigraphy. Thus, unless the sequence has experienced post-depositional 506 disturbance, the luminescence profile should exhibit increasing signal intensities with 
507 depth, commensurate with increasing age as implied by the stratigraphic principle of

508

509

510

511

512

513

514

515

516

517

518

519

520

521

522

523

524

525

526

527

528

529 superposition. Figure $3 a$ is an example of a relatively homogenous sequence in terms of grain size, mineralogy and, to a large extent, dose rate that was deposited over time in a postglacial aeolian dune in Alberta, Canada (Munyikwa et al., 2012). Both the IRSL and post-IR blue OSL signals (CW mode) increase gradually with depth. However, the IRSL/post-IR blue OSL ratio is relatively uniform down the profile, suggesting minimal variation in mineralogy. This indicates that burial age is the most important determinant of signal intensity. In the example depicted in Figure 3a, the aeolian dune sediment was well bleached prior to burial as it shows gradual growth in signal intensity from the top downwards; with the slope on the signal-depth progression providing some insight on the sedimentation rate. Figure 3b reported by Portenga et al. (2016) shows a similar trend. In this instance, fine-grained sediments deposited by fluvial processes in a swampy meadow wetland environment in southeastern Australia show IRSL and post-IR blue OSL signals (CW mode) that increase gradually with depth. The shallow and low-energy environment is thought to have allowed complete bleaching of the sediment prior to burial (Portenga et al., 2016). Hence, since the sediment is assumed to have a common provenance upstream, burial age is also thought to be the dominant influence on signal intensity. Comparable results were reported in similar fluvial deposits from the same region by Muñoz-Salinas et al. (2014). In some settings, sediments are deposited relatively quickly so that the age difference between sediment at the base and at the top of the sequence is minimal. In such cases, the luminescence profiles exhibit signal intensities that are relatively constant throughout the depositional column as in Figure 4 recorded (CW mode) for a coastal dune at Holkham in Norfolk County, UK (Bateman et al., 2015). 
[Insert Figure 3]

534

539 There are cases where poorly bleached or increasingly older sediment can be emplaced 540 above younger deposits. Such profiles would exhibit signals that increase in intensity up 541 the profile, which would be an inverted form of Figure 3. Studies that have reported such 542 inverted profiles include work by Sanderson and Murphy (2010) where multi-wave 543 tsunami events in Thailand eroded increasingly older sediment and deposited it onshore 544 without any significant signal zeroing. Similarly, inverted signal distribution was noted by 545 Sanderson and Murphy (2010) in Italy where what are thought to be excavation tailings 546 were used as backfill overlying a lower archaeological fill after the abandonment of a 547 Neolithic enclosure ditch (Figure 5a). Sanderson and Murphy (2010) suggest that the 548 higher signal intensities (CW mode) in the upper fill indicate that the back filling may have 549 been rapid such that limited signal zeroing occurred. Thus, the upper fill likely contains 550 sediment of mixed age. Luminescence dating using standard protocols of samples from 551 the upper fill and lower fill returned ages that suggest a slightly older age for the upper 552 fill, consistent with the trend from the portable reader signals (Figure 5a). Higher 
553 IRSL/post-IR OSL ratios in the upper fill are also thought to indicate a higher feldspar

554 content because the degree of weathering is not as intense as in the lower fill. Hence,

555 overall, higher portable reader signals in this case were influenced by a combination of

556 factors including degree of signal resetting (and time since last exposure) and feldspar

557 content. Depletion ratios were reported to be uniform throughout the sequence,

558 suggesting that color or grain size did not have major influence on the signal intensities.

559 In south-eastern Australia, Muñoz-Salinas et al. (2014) and Portenga et al. (2016)

560 reported a more or less similar trend where a finer grained swampy meadow (SM) deposit

561 was overlain by a coarser post (European) settlement alluvium (PSA). Portable

562 luminescence signals obtained (CW mode) showed that signal intensities in the SM unit

563 started at very low levels in the upper part and increased steadily down the unit (Figure

$5645 b)$. In the overlying PSA unit, however, the signals were several orders of magnitude

565 higher and displayed much scatter. Measurement with a field dosimeter showed that there

566 were no significant differences in dose rates between the SM and PSA units. Hence,

567 Muñoz-Salinas et al. (2014) concluded that higher signals in the PSA unit likely resulted

568 from poor bleaching of the sediment at the time of deposition and also because there

569 were differences in mineralogy between the SM and PSA units. Poor bleaching was

570 thought to be related to grain size as well as to transport mechanisms. Coarser grains

571 that are transported under turbid conditions are less likely to be reset by daylight

572 compared to finer grains that were deposited by slow moving dilute flow. Overall, Munoz-

573 Salinas et al. (2014) concluded that, unlike the PSA alluvium, SM deposits were

574 appropriate for dating using regular OSL protocols. Samples retrieved from depths of 103 
$575 \mathrm{~cm}$ and $163 \mathrm{~cm}$ returned ages of ca. 2.4 and 5.2 ka respectively, consistent with the higher 576 portable reader signals with increasing depth.

577

578

579 580

581

582 Luminescence profiling can also effectively delineate depositional units of different age 583 584 585 586 587 588 589 590 591 592 593 594 595 596 597
[Insert Figure 5] within a given depositional sequence. As in all profiling studies, the analysis would ideally require signals to be normalized for the effects of variables such as dose rate or sample size prior to comparing the signals for age variation down the profile. For instance, sample aliquots could be weighed accurately prior to conducting luminescence measurements and dose rates could be determined at sampling positions using a portable gamma ray spectrometer. Normalizing the signals for dose rate and sample size would then enable a profile with a time-dominant signature to be obtained, unless other variables such as mineralogical changes or inadequate bleaching are also involved. In a profile that features signals with time-dominant variations, units of different age would be distinguishable as segments of the profile separated by sudden increases in signal intensities. Accordingly, where unconformities or extended gaps in depositional chronology occur, luminescence profiles should display abrupt changes in signal intensities across the uniformities. Multiple profiling studies have been performed with the primary aim of identifying distinct stratigraphic units in depositional sequences. For instance, Muñoz-Salinas et al. (2011), identified a major unconformity in fluvial sediments from an abandoned channel in the 
598 Mekong Delta in Cambodia using this approach (Figure 6a). Visual assessment had 599 identified four depositional units (I-IV) based on color. However, luminescence profiling 600 using the portable reader showed that units I and II in the upper part had relatively similar 601 signal intensities but were significantly different from units III and IV which were both 602 characterized by higher signals and had much higher scatter. A good linear correlation of 603 the signal intensities in units I and II was deemed to indicate that the units were well604 bleached at the time of deposition. The scatter in units III and IV was thought to be a 605 product of bioturbation, given the pervasive presence of worm burrows. Though grain size 606 in unit I was notably coarser than in unit II, the grain size in units III and IV was relatively 607 similar to that in unit II. Hence, grain size did not appear to be the main reason for the 608 difference in signal intensities between units I/II and III/IV. Magnetic susceptibility 609 measurements showed that units III and IV had significantly different susceptibility 610 compared to units $\mathrm{I} / \mathrm{II}$, pointing to a difference in mineralogy and provenance. 611 Furthermore, radiocarbon dating of invertebrate shells from units I and II returned modern 612 ages, which accords with the very low signal intensities. From unit III, however, organic 613 matter returned ages of $5.9 \mathrm{ka}$ and $6.3 \mathrm{ka}$ while from unit IV, an age of 5.7 ka was 614 obtained. Hence, the differences in signal intensities (CW mode) between I/II and III/IV 615 was largely deemed to result from differences in age, though mineralogy may have also 616 had some influence. In Mexico, an interface between two lahars from 1997 and 2001 617 generated by eruptions of the Popocatépetl volcano was also identified using a similar 618 method (Muñoz-Salinas et al., 2011). Because of the young ages of the lahars, portable 619 luminescence profiling of the deposits returned relatively low signals. However, signals 620 from the 2001 deposits were relatively uniform down the profile whereas the 1997 lahar 
621 fluctuated significantly. Muñoz-Salinas et al. (2011) suggested that the differences in the 622 signals was a product of differences in the provenance of the two lahars. The 2001 lahar 623 contains homogenous sediment from a single source whereas the 1997 lahar comprises 624 heterogeneous material from multiple sources. As a result, the differences in 625 luminescence characteristics down each unit enabled the transition between the two units 626 to be delineated.

Luminescence profiling using a portable OSL reader also enabled Rother et al. (2019) to 629 identify a major unconformity between Eemian penultimate interglacial deposits and late 630 glacial cover sands in northeastern Germany (Figure 6b). Deposits at the site were 631 classified into 6 main units. Unit 1 at the base was fine sand (possibly glaciofluvial) that 632 overlay till. Above the basal sand unit was peat that constituted unit 2 . Units $3-5$ overlay 633 the peat and comprised fine-grained laminated lacustrine sediment thought to have been 634 deposited in a depression in dead ice. Overlying the lacustrine deposits, Unit 6 comprised 635 poorly sorted medium-grained sand containing some larger clasts as well as ventifacts. 636 Luminescence profiling (CW mode) showed two main segments. Signal intensities for unit 6376 were fairly consistent throughout the unit. Across the boundary, into units $1-5$, signal 638 intensities increase by 2 orders of magnitude, which pointed to a major unconformity 639 (Figure 6b). While mineralogy, grain size, and dose rate could be possible contributors to 640 the differences in signal intensity, depletion ratios for IRSL and blue OSL were relatively 641 uniform throughout the entire section, showing that sediment bulk properties were not a 642 factor. Also, importantly, OSL dating of 4 samples from unit 6 returned a weighted mean 643 age of $14.2 \pm 0.5 \mathrm{ka}$ while ${ }^{320} \mathrm{Th} / \mathrm{U}$ dating of samples from the lacustrine sediment returned 
644 a corrected age of $118 \pm 7 / 6$ ka and $114 \pm 6 / 5 \mathrm{ka}$. Hence, in this instance, a major 645 influence for the differences in signal intensity was confirmed to be burial age. Rother et 646 al. (2019) concluded that the lacustrine deposits (units 2-5) were deposited during the 647 Eemian interglacial while unit 6 comprised periglacial cover sands deposited by aeolian 648 processes towards the end of the last glacial period.

649

650 Other studies that have employed luminescence profiling to identify discrete units in 651 depositional sequences include work by Munyikwa et al. (2012), Muñoz-Salinas et al. 652 (2013; 2014), Mills et al. (2014), Palamakumbura et al. (2016), Portenga and Bishop 653 (2016), Portenga et al. (2016), Kinnaird et al. (2017), Porat et al. (2019) and Rother et al. 654 (2019). In many cases the differences in the signals have been related to either the 655 depositional age or to the bleaching that occurred prior to burial. Bleaching characteristics 656 are also usually related to the sediment transport and depositional mechanisms.

657

658

659

[Insert Figure 6]

660

661

662 Table 2 outlines luminescence studies that have employed profiling as part of efforts to 663 contextualize the stratigraphy at respective depositional sites. As indicated earlier, in all 664 instances, the portable OSL reader used is the instrument developed by SUERC. For 665 discussion purposes the studies are grouped by environment of deposition, including 666 coastal, fluvial, aeolian, glaciolacustrine, and offshore marine settings. While the 
667 environments may differ, the applications and techniques used in the studies are largely 668 similar. In most studies, the main purpose for employing profiling has been to provide 669 insight into the environment of deposition and to inform sample collection for 670 luminescence dating using standard protocols. Hence, the identification of units that 671 appear to have been well-zeroed before burial is the objective of many studies.

672

673 Some studies combine observations in the field with subsequent laboratory 674 characterization (cf. Burbidge et al., 2007) to 'calibrate' the field profiles, and extend the 675 sediment chronologies into the adjacent stratigraphies (e.g. Kinnard et al., 2017, 2019); 676 or, alternatively, by interpreting signal intensities in light of OSL ages (Porat et al., 2019). 677 Age approximation using portable OSL signals is discussed in greater detail later in this 678 paper.

679 680 [Insert Table 2]

684 From the range of studies outlined in Table 2, it is evident that there is a broad range of 685 settings in which the portable OSL can be applied. The only major requirements are that 686 the deposits being analyzed should contain a dosimeter (normally quartz or feldspar) that 687 was emplaced by geomorphic processes in both space and time. However, there are 688 some environments that are particularly amenable to profiling using the portable OSL 689 reader. Ideally, the sequences being investigated should have grain sizes, mineralogy, 
690 luminescence sensitivity and dose rates that are relatively consistent down the profile

691 such that only burial age is the major determinant of signal intensity. Sediments that were

692 zeroed effectively prior to burial are also preferable as it provides a temporal datum above

693 which stored dose can accumulate following deposition. As with conventional OSL dating,

694 aeolian deposits are particularly suitable for profiling because aeolian deposits are often

695 well-bleached at deposition and wind transport sorts grains into narrow size ranges such

696 as dune sand, or silt-sized loess. Fluvial deposits, on the other hand, are not always well-

697 bleached and sediment grain size ranges can be more variable (e.g. Rittenour, 2008;

698 Cunningham and Wallinga, 2012). With glacial deposits, bleaching is even more limited

699 (e.g. King et al., 2014), though outwash sands would offer the best opportunity for profiling 700 in such environments.

701

702

703

4. INTERPRETING DEPOSITIONAL HISTORIES USING LUMINESCENCE SIGNALS

704

705

As highlighted above, complete zeroing of dose stored in sediment is not always possible

706

before burial. Different sediment transport mechanisms are often associated with

707 bleaching opportunities that are dissimilar. For effective bleaching to occur, sediment

708 should be exposed to adequate sunlight (or heating, in the case of baked sediments).

709 High intensity processes such as turbidity currents or debris flows do not allow adequate

710 time for zeroing (e.g. Muñoz-Salinas et al., 2017a, 2017b, 2018) whereas gradual

711 processes at the earth's surface, such as subaerial transport of sediments through

712 saltation that occurs with aeolian dune sediments (e.g. Singhvi and Porat, 2008), offer the 
713 best opportunities. In this section, studies that have explored bleaching characteristics of 714 different environments using portable OSL readers are examined. The studies are 715 grouped into two main categories. The first looks at studies where the extent of signal 716 resetting associated with some depositional environments were investigated (e.g. King et 717 al., 2014; Muñoz-Salinas et al., 2018). The second looks at studies where bleaching 718 characteristics were used to elucidate geomorphic processes (Stang et al., 2012; Castillo 719 et al., 2014; Muñoz-Salinas, 2017b, 2018). The use of luminescence signals as sediment 720 tracers is also examined briefly.

4.1 Examining bleaching characteristics of depositional environments

The analysis of inherited luminescence signal characteristics of modern sedimentary environments using a portable OSL reader enables the identification of optimal bleaching conditions for a given depositional system. Insight into bleaching characteristics acquired 728 from such investigations facilitates informed targeting when sampling for conventional 729 OSL dating is directed at fossil landforms. For instance, Muñoz-Salinas et al. (2017a) 730 investigated bleaching in sediments younger than 2 ka from fluvial, coastal, and volcanic 731 environments from Mexico using a portable OSL reader. Samples were analyzed using 732 IRSL and blue OSL stimulation in CW mode. As expected, the results generally showed 733 that sediments transported by debris flows had the highest residual signals since the 734 opportunity for bleaching was limited. It was also noted that IRSL signals from volcanic 735 ash and coastal deposits (beach and dune sand) were characterized by low scatter. The 
736 blue OSL signal, however, had significant scatter for both coastal and volcanic sediments.

737 For coastal deposits, Munoz-Salinas et al. (2017a) attributed the scatter to charge transfer

738

739

740

741

742

743

744

745

746

747

748

749

750

751

752

753

754

755

756

757

758

whereas for volcanic deposits, low quartz sensitivity was suspected. The fluvial deposit exhibited a high degree of scatter with both IRSL and blue OSL stimulation, possibly from sediment mixing.

A more focused study that examined bleaching mechanisms was conducted by King et al. (2014) who investigated catchment-scale variability in residual signals of modern glacial sediments in Jostedalen, southern Norway. Samples were collected from shallow depths in glaciofluvial bars including braided bars and side-attached bars from four different catchments. Additional samples were also collected from subglacial, paraglacial, and avalanche deposits that served as sources for the glaciofluvial sediments. Sample measurement was performed using a portable OSL reader ( $\mathrm{CW}$ mode) and, overall, residual signals in bar environments fell as the glaciofluvial sediment was carried further from the ice margin or from the sediment source (e.g. Figure 7). Slope failure deposits and sheet wash deposits had some of the highest residuals since such rapid depositional processes offered limited opportunity for exposure to sunlight (King et al., 2014). For glaciofluvial deposits, residual signal intensities also depended on morphological aspects of the depositional feature. For instance, braid-bar-head deposits generally had the highest residual signal intensities while braid-bar-mid, braid-bar-tails and side-attached bars had some of the lowest. For some sites, settings where poorly bleached sediment was continuously added to the sediment load as it moved downstream resulted in a high degree of luminescence signal variability. Thus, the results confirmed that high magnitude 
759 events of low frequency such as turbulent flows had poor sediment bleaching 760 opportunities whereas low magnitude events that occurred with a higher frequency, such 761 as deposition in braid-bars-mid and tails, offered greater chances for bleaching (King et 762 al., 2014).

763

764 Overall, the studies by King et al. (2014) and Munoz-Salinas et al. (2017a) demonstrated 765 the utility of the portable luminescence reader to rapidly characterise bleaching of 766 sediment in a range of modern depositional settings. Observations from such studies can 767 inform sampling strategies in conventional OSL dating of fossil landforms. [Insert Figure 7]

Beyond screening samples for conventional OSL dating, luminescence signal 776 characteristics acquired using a portable OSL reader have also been used to infer 777 geomorphic processes associated with depositional sequences. In a study by Stang et al. 778 (2012), the instrument was used to assess soil mixing processes and rates at a site in the 779 San Gabriel Mountains, California. The study had noted that in order to understand 780 processes that influence rates of weathering and erosion at some scales, there was a 781 need to evaluate the relative significance of mechanisms by which clastic particles are 
782 translocated within soil profiles and their rates. Samples were collected at constant 783 intervals from three soil profiles on hillslopes within a $100 \mathrm{~m}$ radius. Using the portable 784 OSL reader, IRSL and post-IR blue OSL signals were obtained from each sample in CW 785 mode. To allow a measurement of relative time, an IRSL growth curve was constructed 786 using a conventional OSL reader and select bulk samples from the study area. The growth 787 curve was used to convert dose values into 'effective age' estimates. Figure 8 outlines 788 plots of effective age versus depth for the three monoliths sampled in the study. Low 789 'effective ages' were interpreted as denoting episodes during which sediment was close 790 to the surface such that the grains were bleached by sunlight or heated by wildfires before 791 being subsequently translocated downward.

797 In another study that aimed to unravel sediment transport mechanisms, Castillo et al. 798 (2014) used signal intensities acquired using a portable OSL reader to compare rates of 799 erosion that followed late Cenozoic differential tectonic uplift of the Jalisco Block in 800 western Mexico. The results showed that high IRSL signal intensities were recorded for 801 samples from the northern sector of the Jalisco Block while lower intensities were noticed 802 in the southwest. Though dose rates were not provided, Castillo et al. (2014) argued that 803 the high signal intensities from the northern sector resulted from higher rates of uplift that 804 contributed a higher sediment load while lowering opportunities for bleaching. Lower rates 
805 of uplift in the southern sector, on the other hand, produced lower sediment loads and 806 gentler flows which were associated with greater chances of bleaching. Similar results 807 were reported by Munoz-Salinas et al (2017a) who examined sediment in rivers that cross 808 two major faults in the Sierra De Juárez mountains in southern Mexico. Here, results 809 showed that inherited portable OSL signals were higher in basins with steeper slopes 810 compared to those with more gentle relief. Hence, the signals from steeper slopes were 811 consistent with sediment transported in debris flows or in hyper-concentrated flows where 812 turbidity was significant.

814 Mechanisms of sediment transport in other fluvial settings were examined by Muñoz815 Salinas et al. (2018) who compared rates of erosion between areas in central Mexico 816 under conservation with those where natural vegetation was allowed to grow. In this study 817 too, streams characterized by hyper-concentrated or debris flows did not exhibit much 818 bleaching. Additionally, streams where fresh sediment that is poorly bleached was 819 continuously incorporated into the stream exhibited luminescence intensities that 820 increased downstream (Figure 9). Overall, the results showed that conservation efforts in 821 the area were unsuccessful as they caused landscape instability compared to areas with 822 natural vegetation (Munoz-Salinas et al., 2018). In the Wadi Suf in Jordan, Lichtenberger 823 et al. (2019) analyzed three profiles along the Chrysorrhoas River and noted that 824 luminescence signal intensities increased downstream. However, in this instance the 825 higher signals were attributed to increase in luminescence sensitivity following repeated 826 cycles of deposition, erosion and transportation (Lichtenberger et al., 2019). 
828 Table 3 summarizes studies that have explored sediment bleaching using a portable OSL

829 reader. The application of luminescence as a sediment tracer and provenance tool has

830 been reviewed by Gray et al. (2019). Though the review does not focus exclusively on

831 the use of portable readers, it highlights areas where luminescence could be used to 832 identify source lithologies and transport mechanisms based on luminescence 833 characteristics.

834

835

836

[Insert Figure 9]

837

838

839

840

[Insert Table 3]

841

842

843

\section{APPROXIMATING SEDIMENT BURIAL AGE USING A PORTABLE OSL READER}

844

845 An area that has seen some emerging applications in efforts to expand the utility of 846 portable OSL readers has been the calibration of portable signals so that they can be 847 used for approximating numerical ages, as opposed to simply providing relative 848 chronology. A number of approaches have been used, ranging from the application of 849 standardised growth curves (SGCs) generated using normalised portable OSL reader 850 signals to approximate the equivalent dose (e.g. Munyikwa and Brown, 2014), to the 
851 construction of calibration curves that use samples whose ages have been determined 852 using standard luminescence dating protocols and have also had their signals measured 853 using portable luminescence readers (e.g. Stone et al., 2015, 2019). While the methods 854 have been applied primarily to inland aeolian deposits (e.g. Munyikwa and Brown, 2014; 855 Stone et al., 2015; 2019), age estimation using portable OSL readers has also been 856 extended to coastal sediments (e.g. Brill et al., 2016), and to wetland deposits that 857 comprise aeolian and fluvial sediments (e.g. Gray et al., 2018). With wetland deposits, 858 the possibility of sediment mixing and partial bleaching that is associated with such 859 environments appears to introduce significant scatter in the data, likely more than would 860 be expected with well-bleached aeolian deposits. Nonetheless, as range finders that 861 assist targeting of sampling and further follow-up, the age approximations provide a useful 862 aid in luminescence studies. Each of these approaches is discussed below.

Modern versions of SGCs were proposed by Roberts and Duller (2004) as an approach 867 that could truncate age determination procedures in luminescence dating assessment, 868 especially when conducting reconnaissance studies. With SGCs, luminescence growth 869 curves are constructed using normalized signals and once a curve is constructed, the $D_{e}$ 870 of a natural sample of unknown age is determined by simply acquiring a normalised 871 natural signal which is then compared with the SGC to derive a corresponding $D_{e}$. In 872 essence, the use of an SGC negates the construction of a unique growth curve for each 873 sample, which is the standard procedure in luminescence dating. Typically, SGCs are 
874 appropriate for sediments that share similar luminescence properties. Hence, they are 875 regionally applicable at best.

877 The combination of an SGC with a portable OSL reader that can analyze bulk samples 878 appears to be an attractive concept as it abbreviates the process of age estimating 879 significantly (Munyikwa and Brown, 2014). There are two main challenges that are 880 associated with constructing SGCs using portable OSL reader signals. The first is that a source of artificial radiation is required for constructing the growth curve as well for 882 administering test doses for signal normalization. Since the only portable OSL device used for dating applications so far, the SUERC portable OSL reader, does not have an 884 internal radiation source, external sources are needed (e.g. Sanderson \& Murphy, 2010; 885 Munyikwa and Brown, 2014). Secondly, for those readers that may have an internal 886 source, irradiated samples would need to be heated prior to measurement in order to 887 eliminate charges in unstable traps. However, heating a sample in a portable OSL reader 888 places challenges that would need to be overcome, ranging from excessive power 889 demand to the possibility of combustion of organics in an untreated sample, and 890 condensation of moisture from wet sediment (Roberts et al., 2018). Heating of samples 891 has been conducted outside the reader in some studies (e.g. Munyikwa and Brown, 892 2014), while other OSL readers have the means to minimize the effects of condensation 893 (e.g. Kook et al., 2011). Alternatively, samples could be dried before analyzing but that 894 may detract from the rapidity for which portable OSL readers are known. Figure 10 is an 895 SGC constructed by Munyikwa and Brown (2014) using a portable OSL reader. Test 896 doses for normalisation were applied using an external gamma-ray source. Normalized 
897 signals were then obtained by dividing the regeneration signal $\left(L_{x}\right)$ by the test dose signal $898\left(T_{x}\right)$ and multiplying the quotient by the test dose $\left(T_{d}\right)$. In the saturation exponential used 899 to fit the data, $I$ is the standardised luminescence signal given by dose $D$, the intercept is 900 given by $I_{0}$, and $I_{\max }$ is the upper limit for $I$. The rate at which dosimeter traps are filled 901 determines $D_{0}$ (Munyikwa and Brown, 2014).

Overall, the lack of an internal radiation source in some portable OSL readers means that the construction of SGCs as a rapid mechanism for approximating sample ages may not easily be attainable for researchers without access to an external radiation source. The lack of heating capability may also be an additional constraint to some. Nonetheless,

911 future technical advancements in the design of portable OSL readers may render this 912 possible. Notably, Roberts et al. (2018) have proposed three different methods for 913 approximating $D_{e}$ that do not require heating of samples and could be used with portable 914 OSL readers. The first approach requires the derivation of a correction factor that is 915 obtained from comparing $D_{e}$ values determined using heating with those obtained using 916 no heating. The second approach attempts to subtract the effects of the $110^{\circ} \mathrm{C} \mathrm{TL}$ peak 917 and other unstable traps from the unheated quartz signal through component fitting. The 918 third method involves administering a small beta dose to a sample before measuring the 919 natural signal. The beta dose fills the $110^{\circ} \mathrm{C}$ TL peak such that the measurement obtained 
920 would be comparable to a regeneration measurement that has not been heated and,

921 hence, has the same peak. While these approaches have been tested on pure quartz

922 separates using Ris $\varnothing$ TL/OSL-DA-12 or 20 systems, they still need to be tested using

923 bulk samples, and portable OSL readers for that matter. Thus, overall, the approaches

924 provide scope for future development of the technique going forward.

925

926

927 5.2. Regression curves that compare known sample ages and their portable OSL reader 928 signals

929

930

An approach for approximating sample ages using portable OSL reader signals that 931 requires no irradiations nor preheating was initially introduced by Stone et al. (2015). The

932 procedure constructed a calibration curve by plotting sample ages that had previously 933 been determined using standard OSL protocols against luminescence signals obtained

934 from bulk aliquots of the samples using a portable OSL reader. In the study, a total of 16 935 previously dated samples from the Namib Sand Sea in southern Africa, ranging in age 936 from the last interglacial to the Late Holocene (e.g. Stone et al. 2010) were analysed using 937 a portable OSL reader in CW mode. Importantly, dose rates, mineralogy and bleaching 938 characteristics for the sediments were fairly uniform and burial age rather than grain size 939 variation, variations in quartz to feldspar ratio, or sensitivity, was the main factor 940 influencing luminescence signal intensity. A regression curve of post-IR OSL signals 941 versus the standard OSL ages showed a good fit. To test the calibration curve, signals 942 from samples that had been excluded in constructing the curve were used as input values 
943 for the regression function determined on known ages samples. Results confirmed that 944 the curve could be used to assign numerical age approximations to samples from the 945 study area.

947 In a subsequent study that expanded on the earlier work, Stone et al. (2019) constructed 948 regression curves using portable OSL signals from 144 previously dated aeolian dune 949 samples from southern Africa. As in the earlier study, sample ages ranged from the last 950 interglacial to the late Holocene. Apart from providing a more robust dataset, the study 951 also investigated whether a single generic regression curve would suffice for the entire 952 region or if region-specific curves were required. When results from individual sites were 953 plotted to show the variation of portable reader signals and OSL ages with depth, there 954 was good correlation between the three variables, affirming the capability of the portable 955 OSL reader to measure relative age with depth. Plotting post-IR blue OSL signals against 956 OSL ages for the 144 samples (Fig 11) showed that the results could be fitted into four 957 main regional regression models. The regional groupings were largely thought to be an 958 artifact of differences in feldspar to quartz ratio, luminescence sensitivity and local dose 959 960 major effect on the signals (Stone et al., 2019).

961

962 Overall, the attractiveness of the approach by Stone et al. $(2015 ; 2019)$ is that, once the 963 calibration curve had been established, all that is required to approximate the age of a 964 sample of unknown age is the bulk signal measured using the portable OSL reader. The 965 modest costs associated with the procedure is such that large numbers of age estimates 
966 can be obtained using minimal resources, allowing for high resolution depositional 967 frameworks to be developed. Furthermore, the rapid nature of the approach facilitates 968 effective targeting during the sample collection stage when sampling for full-fledged 969 dating (Stone et al., 2019).

970 971

972 [Insert Figure 11]

973

974

975 Similar to the method employed by Stone et al. $(2015 ; 2019)$, Porat et al. (2019) calibrated 976 portable OSL reader signals to assign a numerical time scale to the geomorphic evolution 977 of archaeological terraces in the Judean Highlands of Israel. Samples were collected at 978 constant intervals from pits excavated in the terraces after which portable reader 979 measurements were made to determine post IR blue OSL signals (CW mode). 980 Additionally, samples were collected from positions that had been sampled for portable 981 reader measurements and dated using conventional OSL protocols (Figure 12a). A 982 comparison of standard OSL ages and associated portable OSL signals showed good 983 correlation, allowing a linear regression function to be derived (Figure 12b). Thus, the 984 function was used to convert the rest of the portable luminescence signals from the 985 excavation pit to numerical age approximations. 
991 While the calibration of portable OSL signals using known ages by Stone et al. (2015;

992 2019) examined aeolian depositional systems that were largely homogenous, the 993 approach was extended to heterogenous depositional systems by Gray et al. (2018) who 994 examined complex paleowetland deposits from the Las Vegas Valley in Nevada. The 995 study aimed to ascertain if luminescence signal intensities of the interbedded aeolian and 996 fluvial deposits had a strong age component or if the complex depositional mechanisms 997 and provenance played a greater influence. Samples were collected from units with well998 established radiocarbon and OSL ages ranging from 573 -11 ka (Gray et al., 2018) and 999 analyzed using IRSL and blue OSL (CW mode). Regression models of the portable OSL 1000 signals against the ages showed that the data could be fitted with a quadratic equation 1001 though some scatter was observed and this possibly arose from partial bleaching in the 1002 fluvial sediment, variations in sediment source lithology, as well as differences in 1003 sensitivity. Sieving the sediment and removing magnetic minerals appeared to reduce the 1004 scatter significantly (Figure 13). Overall, the study showed that, even in complex 1005 depositional systems, age can be a major influence on paleodose levels such that burial 1006 ages can be approximated using portable OSL reader signals (Gray et al., 2018). 
1012 Table 4 outlines some studies that have employed portable OSL readers to approximate 1013 sample ages. This is an area that is increasingly receiving attention and is likely to 1014 produce significant new developments in the near future.

1015

1016

1017

1018

1019

1020

1021

1022

1023

1024

1025

1026

1027

1028

1029

1030

1031

1032

1033

1034

[Insert Table 4]

\section{CONCLUSION AND FURTHER OUTLOOK}

Functional portable OSL readers that can be used in geomorphological applications have only been in existence for about a decade. However, a sizeable body of literature has accumulated during this period. Measurements using the instrument can be made on unprocessed samples, making the technique less resource-intensive than regular luminescence dating protocols. Additionally, the instrument can be brought closer to the field site if needed, enabling near real-time data to be obtained during fieldwork.

Luminescence signal intensities of sediments are influenced by variables that include burial age, luminescence sensitivity, dose rates and mineralogy and other bulk properties. Thus, in many instances the measurements performed using the portable OSL reader allow stratigraphic features that are not visually discernible to be elucidated including cryptostratigraphic features and mixed horizons (e.g. Sanderson and Murphy, 2010). Overall, the tool can offer significant advantages when used to provide reconnaissance data as part of a luminescence dating study. The prospects of analyzing larger numbers 
1035 of samples in less time and at lower cost allows for high-resolution chronostratigraphic 1036 frameworks to be developed, which aids sample selection for conventional luminescence 1037 dating. Additionally, improved chronological insight of the stratigraphic sequences 1038 enables geomorphic contexts to be elucidated, which helps in the interpretation of the 1039 data. While studies completed to date can generally be grouped into three main 1040 categories: generation of luminescence profiles of depositional sequences, analyzing 1041 bleaching properties of sediments, and approximating ages of clastic sediments, many of 1042 the studies overlap more than one area. An overarching characteristic of the studies is 1043 that the portable OSL reader lends a rapid semi-quantitative approach to the analysis of 1044 stratigraphic sequences that would otherwise not be possible without the device.

1046 Applications in which the portable OSL reader has been employed to date show that there 1047 is a broad range of geomorphic settings in which the instrument can be used. Clastic 1048 sediments of late Quaternary age containing either quartz or feldspar offer the best 1049 opportunities for analysis using the device. The instrument functions optimally to provide 1050 chronological information in settings where the sediment has been well bleached prior to 1051 the last burial event and where variables such as mineralogy, dose rates, and 1052 luminescence sensitivity are relatively homogenous. In more complex depositional 1053 environments, however, the utility of the portable OSL reader can be constrained as a 1054 chronostratigraphic tool, though it could be used for sediment tracing. This includes 1055 settings where dose rates vary significantly between units, or where differences exist in 1056 lithology, provenance or mineralogy such as in mixed clay sand and gravel units or in 1057 glacial and fluvial environments. Accordingly, depositional environments in which the 
1058 device has been used range from aeolian (e.g. Munyikwa et al., 2012; Bateman et al., 1059 2015) to fluvial (e.g. Muñoz-Salinas et al., 2013; Kinnaird et al., 2015) and glacial settings

1060 (King et al., 2014). In some studies, complex sequences resulting from interactions 1061 between aeolian and fluvial (e.g. Gray et al., 2018), and marine processes have also been 1062 investigated (e.g. Palamakumbura et al., 2016; Sanderson \& Kinnaird 2019) with varying 1063 degrees of success. Furthermore, the applications of the portable OSL reader span from 1064 purely geomorphological investigations (Bateman et al., 2015) to geoarchaeological and 1065 archaeological studies (e.g. Kinnaird et al., 2017; Porat et al., 2019). In essence, the utility 1066 of the instrument is multifaceted.

1068 Apart from the widening range of applications in which the portable OSL reader have 1069 been used, the increasing body of work has notably been accompanied by greater 1070 sophistication in the type of studies being undertaken. Hopefully, going forward, this will 1071 be matched by further developments in the portable OSL reader hardware. The group at 1072 SUERC has recently introduced a double IR system that conducts stimulation using 89 $1073 \mathrm{~nm}$ and $940 \mathrm{~nm}$ wavelength sources. This setup results in ultra-high sensitivity for very 1074 young sediments which enables more recent events to be analyzed. It has also been 1075 suggested to move beyond simple profiling measurements which are largely one- or two1076 dimensional at best to generating three-dimensional visualizations of the 1077 chronostratigraphy (e.g. Verust et al., 2019). In particular, three-dimensional models 1078 could be advantageous for analyzing aeolian sequences that form planar bedforms, which 1079 includes many fossil dune sediments. A greater understanding of such bedforms and the 1080 associated temporal structure would be invaluable in the interpretation of luminescence 
1081 chronologies because the ages are usually obtained as point data. Three-dimensional 1082 visualization of a depositional unit would permit estimation of its magnitude, which could 1083 be related to the intensity of the environmental or climatic event during which the sediment 1084 accumulated as well as sediment supply. The spatial dimensions of the unit could also be 1085 used to infer direction of movement of an aeolian dune, which can be used to elucidate 1086 paleowind directions.

1088 An area that has potential for growth is in the interpretation of data acquired using the 1089 portable OSL reader. To date, the interpretation of the data has largely involved univariate 1090 analysis, with only a single aspect being examined at a time. However, in many cases 1091 this is a simplification of systems that are known to be complex. Hence, it would be helpful 1092 to start incorporating portable OSL proxy variables in multivariate analyses that examine 1093 a broad range of other geomorphological variables. As indicated above, portable OSL 1094 reader signal intensities are influenced by a range of factors apart from burial age (e.g. 1095 dose rates, luminescence sensitivity, mineralogy, etc.). If these factors could be 1096 dynamically included in the analysis, the results would allow reconstructions that are 1097 cognizant of the interrelationships found in natural depositional systems. Additionally, it 1098 would make it possible to analyze geomorphic environments that are more complex.

1100 Research in multiple other developmental areas are also in progress at the SUERC lab 1101 on topics ranging from the use of pulsed stimulation to analyze post stimulation 1102 phosphorescence and running sequences that explore time-width signal variation. 
1103 However, as outlined in the introduction, these topics would be more suitable for a more 1104 dedicated review paper.

1106 Finally, efforts to determine $D_{e}$ using the portable reader have demonstrated the potential 1107 to expand the utility of the instrument significantly. The studies have ranged from the 1108 construction of SGCs using normalized portable OSL signals (e.g. Munyikwa and Brown, 1109 2014) to calibrating portable OSL signals using data from ages dated using standard 1110 protocols (e.g. Stone et al., 2015, 2019; Gray et al., 2018). These efforts could also benefit 1111 from the development of protocols that can be used for estimating $D_{e}$ without sediment 1112 heating, as proposed by Roberts et al. (2018). If all these techniques were to be 1113 successfully refined and made functional, they would provide practitioners with a range 1114 of approaches that could routinely be used to generate approximate absolute ages using 1115 portable OSL readers, a prospect many would find invaluable. Negating sediment heating 1116 during measurement may not be useful to practitioners with portable OSL readers that 1117 do not have internal irradiators as these are required for determining $D_{e}$. However, for 1118 those with irradiators that have internal X-ray sources (e.g. the instruments developed by 1119 Takeuchi et al. (2008) and by Kook et al. (2011)), the development would certainly be 1120 consequential.

1122 Overall, given the progress made to date, the application and utility of the portable OSL 1123 reader is set to expand. Its utility is gradually being affirmed by the broad diversity of 1124 geomorphic settings in which the instrument is being applied. It is possible to foresee a 
1125 time in the future when the device will be a standard tool in many studies that look at late

1126 Quaternary landscape evolution.

\section{REFERENCES}

Aitken, M.J. (1998) An introduction to optical dating. Oxford: Oxford University Press.

1132 Ankjaergaard, C., Jain, M., Thomsen, K.J. and Murray, A.S. (2015) Optimising the 1133 separation of quartz and feldspar optically simulated luminescence using pulsed 1134 excitation. Radiation Measurements, 45, 778-785.

1136 Bailey, R.M. (1998) Depletion of quartz OSL signal using low photon energy stimulation. 1137 Ancient TL, 16, 33-36.

1139 Bateman, M.D., Rushby, G., Stein, S., Ashurst, R.A., Stevenson, D., Jones, J.M.and 1140 Gehrels, W.R. (2018) Can sand dunes be used to study historic storm events? Earth 1141 Surface Processes and Landforms, 43, 779-790.

1143 Bateman, M.D., Stein, S, Ashurst, R.A. and Selby, K. (2015) Instant luminescence 1144 chronologies? High resolution luminescence profiles using a portable luminescence 1145 reader. Quaternary Geochronology, 30, 141-146. 
1147 Bishop, P., Muñoz-Salinas, E., Mackenzie, A.B., Pulford, I. and Mckibbin, J. (2010) The 1148 character, volume and implications of sediment impounded in mill dams in Scotland: The 1149 case of the Baldernock Mill dam in East Dunbartonshire. Earth and Environmental 1150 Science Transactions of the Royal Society of Edinburgh, 101, 97-110.

1151

1152 Brill, D., Jankaew, K. and Brückner, H. (2016) Towards increasing the spatial resolution 1153 of luminescence chronologies - Portable luminescence reader measurements and 1154 standardized growth curves applied to a beach-ridge plain (Phra Thong, Thailand). 1155 Quaternary Geochronology, 36, 134-147.

1157 Brill, D., May, S.M., Shah-Hosseini, M., Rufer, D., Schmidt, C. and Engel, M. (2017) 1158 Luminescence dating of cyclone-induced washover fans at Point Lefroy (NW Australia). 1159 Quaternary Geochronology, 41, 134-150.

1160

1161 Bristow, C.S., Duller, G.A.T. and Lancaster, N. (2007) Age and dynamics of linear dunes 1162 in the Namib Desert. Geology, 35, 555-558.

1164 Burbidge, C.I., Sanderson, D.C.W., Housely, R.A. and Allsworth Jones, P. (2007) Survey 1165 of Paleolithic sites by luminescence profiling: A case study from Eastern Europe. 1166 Quaternary Geochronology, 2, 290-302.

1168 Carter, J, Cresswell, A.J., Kinnaird, T.C., Carmichael, L.A., Murphy, S. and Sanderson, 1169 D.C.W., 2018. Non-Poisson variations in photomultipliers and implications for 
1170 luminescence dating. Radiation Measurements, 120, 267-273.

\section{1 doi:10.1016/j.radmeas.2018.05.010}

1172

1173 Castillo, M., Muñoz-Salinas, E. and Ferrari, L. (2014) Response of a landscape to

1174 tectonics using channel steepness indices $\left(k_{s n}\right)$ and OSL: A case study from the Jalisco

1175 Block, Western Mexico. Geomorphology, 221, 204-214.

1176

1177 Clark, R.J. and Sanderson, D.C.W., (1994) Photostimulated Luminescence Excitation

1178 Spectroscopy of feldspars and micas, Radiation Measurements, 23, 641-646

1179

1180 Cunningham, A.C. and Wallinga, J. (2012) Realizing the potential of fluvial archives

1181 using robust OSL chronologies. Quaternary Geochronology, 12, 98-106.

1182

1183 Denby, P.M., Bøtter-Jensen, L., Murray, A.S., Thomsen, K.J. and Moska, P. (2006)

1184 Application of pulsed OSL to the separation of the luminescence components from a 1185 mixed quartz/feldspar sample. Radiation Measurements, 41, 774-779.

1187 Duller, G.A.T. (2003) Distinguishing quartz and feldspar in single grain luminescence 1188 measurements. Radiation Measurements, 37, 161-165.

1190 Duller G.A.T. and Bøtter-Jensen, L. (1993) Luminescence from potassium feldspars 1191 stimulated by infrared and green light. Radiation Protection Dosimetry, 47, 683-688. 
1193 Durcan, J.A., Roberts, H.M., Duller, G.A.T. and Alizai, A.H. (2010) Testing the use of 1194 range-finder OSL dating to inform field sampling and laboratory processing strategies. 1195 Quaternary Geochronology, 5, 86-90.

1196

1197 Galloway, R.B. (1994) Comparison of green and infrared stimulated luminescence of 1198 feldspars. Radiation Measurements, 23, 617-620.

1199

1200 Ghilardi, M., Sanderson, D., Kinnaird, T., Bicket, A., Balossino, S., Parisot, J.-C., Hermitte 1201 D., Guibal, F. and Fleury, J.T. (2015) Dating the bridge at Avignon (south France) and 1202 reconstructing the Rhone River fluvial palaeo-landscape in Provence from medieval to 1203 modern times. Journal of Archaeological Science: Reports, 4, 336-354.

1204

1205 Gray H.J., Mahan, S.A., Springer, K.B. and Pigati, J.S. (2018) Examining the relationship 1206 between portable luminescence reader measurements and depositional ages of 1207 paleowetland sediments, Las Vegas Valley, Nevada. Quaternary Geochronology, 48, 80120890.

1210 Gray, H.J., Jain, M., Sawakuchi, A.O., Mahan, S.A. and Tucker, G.E. (2019) 1211 Luminescence as a Sediment Tracer and Provenance Tool. Reviews of Geophysics, 57, 1212 987-1017 (https://doi.org/10.1029/2019RG000646). 
1214 Jain M. and Singhvi, A.K. (2001) Limits of depletion of blue-green light stimulated

1215 luminescence in feldspars: implication for quartz dating. Radiation Measurements, 33,

1216 883-892.

1217

1218 Kappler, C., Kaiser, K., Tanski, P., Klos, F., Fülling, A., Mrotzek, A., Sommer, M. and 1219 Bens, O. (2018) Stratigraphy and age of colluvial deposits indicating Late Holocene soil 1220 erosion in northeastern Germany. Catena, 170, 224-245.

1221

1222 King, G.E., Sanderson, D.C.W., Robinson, R.A.J. and Finch, A.A. (2014) Understanding 1223 processes of sediment beaching in glacial settings using a portable OSL reader.

1224 Boreas, 43, 955-972.

1225

1226 Kinnaird T, Dawson T, Sanderson D. Hamilton D. Cresswell A Rennell R., (2019)

1227 Chronostratigraphy of an Eroding Complex Atlantic Round House, Baile Sear, Scotland,

1228 The Journal of Island and Coastal Archaeology, 14:1, 46-60, DOI:

$1229 \quad 10.1080 / 15564894.2017 .1368744$

1230

1231

1232 luminescence profiling and dating of historic agricultural terraces in Catalonia (Spain).

1233 Journal of Archaeological Science, 78, 66-77.

1234

1235 Kinnaird, T., Dixon, J.E., Robertson, A.H.F., Peltenburg, E. and Sanderson D.C.W.

1236 (2013) Insights on topography development in the Vasilikós and Dhiarizos Valleys, 
1237 Cyprus, from integrated OSL and Landscape Studies. Mediterranean Archaeology and 1238 Archaeometry, 13, 49-62.

1239

1240 Kinnaird, T.C., Sanderson, D.C.W. and Bigelow, G.F. (2015) Feldspar SARA IRSL

1241 dating of very low dose rate aeolian sediments from Sandwick South, Unst, Sheltand.

1242 Quaternary Geochronology, 30, 168-174.

1243

1244 Kinnaird, T.C., Sanderson, D.C.W. and Woodward, N.L. (2012) Applying luminescence 1245 methods to geoarchaeology: a case study from Stronsay, Orkney. Earth and

1246 Environmental Science Transactions of the Royal Society of Edinburgh, 102, 191-200.

1248 Kocurek, G. and Ewing, R.C. (2005) Aaeolian dune field self-organization - implications 1249 for the formation of simple versus complex-dune field patterns. Geomorphology, 72, 941250105.

1251

1252 Kook, M.H., Murray, A.S., Lapp, T., Denby, P.H., Ankjaergaard, C., Thomsen., K.J., 1253 Jain, M., Choi, J.H. and Kim, G.H. (2011) A portable luminescence dating instrument.

1254 Nuclear Instrument and Methods B, 269, $1370-1378$.

1255

1256 Lichtenberger, A., Raja, R., Seland, E.H., Kinnaird, T. and Simpson, I.A. (2019) Urban1257 riverine hinterland synergies in semi-arid environments: millennial-scale change, 1258 adaptations, and environmental responses at Gerasa/Jerash. Journal of Field 1259 Archaeology, 44, 333-35. 
1261 Mills, C., Simpson I. and Adderley, P. (2014) The lead legacy: the relationship between

1262 historical mining, pollution and the post-mining landscape. Landscape History, 35, 47126372.

1264

1265 Muñoz-Salinas, E., Bishop, P., Sanderson, D.C.W., Kinnaird, T. and Zamorano, J.J. 1266 (2011) Interpreting luminescence data from a portable OSL reader: three case studies 1267 in fluvial settings. Earth Surface Processes and Landforms, 36, 651-660.

1269 Muñoz-Salinas, E., Bishop, P., Sanderson, D. and Kinnaird, T. (2014) Using OSL to 1270 assess hypothesis related to the impacts of land use change with the early nineteenth 1271 century arrival of Europeans in south-eastern Australia: an exploratory case study from 1272 Grabben Gullen Creek, New South Wales. Earth Surface Processes and Landforms, 39, $1273 \quad 1576-1586$.

1274

1275 Muñoz-Salinas, E., Castillo, M., Sanderson D. and Kinnaird, T. (2013) Unravelling 1276 paraglacial activity on Sierra de Gredos, Central Spain: a study based on geomorphic 1277 markers, stratigraphy and OSL. Catena, 110, 207-214.

1279 Muñoz-Salinas, E. and Castillo, M. (2018) Assessing conservation practices in 1280 Amalacaxco Gorge (Izta-Popo National Park, Central Mexico) using fallout ${ }^{137} \mathrm{CS}$ and 1281 optically stimulated luminescence (OSL). Journal of Mountain Science, 15, 447-460. 
1283 Muñoz-Salinas, E., Castillo, M. and Arce, J.L. (2017a) OSL signal resetting in young 1284 deposits determined with a pulsed photon-stimulated luminescence (PPSL) unit. Boreas, $128546,325-337$.

1286

1287 Muñoz-Salinas, E., Castillo, M., Caballero L. and Lacan, P. (2017b) Understanding 1288 landscape dynamics of the Sierra de Juarez, southern Mexico: an exploratory approach 1289 using inherited luminescence signals. Journal of South American Earth Sciences, 76, $1290 \quad 208-217$.

1291

1292 Munyikwa, K., Brown, S. and Kitabwala, Z. (2012) Delineating stratigraphic breaks at 1293 the bases of postglacial aeolian duns in central Alberta, Canada using a portable OSL 1294 reader. Earth Surface Processes and Landforms, 37, 1603-1614.

1295

1296 Munyikwa, K. and Brown S. (2014) Rapid equivalent dose estimation for aeolian dune 1297 sands using a portable OSL reader and polymineralic standardised luminescence growth 1298 curves: expedited sample screening for OSL dating. Quaternary Geochronology, 22, 1161299125.

1300

1301 Palamakumbura, R.N, Robertson, A.H.F., Kinnaird, T.C. and Sanderson D.C.W. (2016)

1302 Sedimentary development and correlation of Late Quaternary terraces in the Kyrenia

1303 Range, northern Cyprus, using a combination of sedimentology and optical

1304 luminescence data. International Journal of Earth Sciences, 105, 439-462. 
1306 Píšková, A., Roman, M., Bulínová, M., Pokorný, M., Sanderson, D., Cresswell, A., Lirio, 1307 J.M., Coria, S.H., Nedbalová, L., Lami, A., Musazzi, S., Van de Viyver, Nývlt D. and 1308 Kopalová, K. (2019) Late-Holocene palaeoenvironmental changes at Lake Esmeralda 1309 (Vega Island, Antarctic Peninsula) based on a multi-proxy analysis of laminated lake 1310 sediment. Holocene, 29, 1155-1175.

1311

1312 Poolton, N.R.J., Bøtter-Jensen, L, Wintle, A.G., Jakobsen, J., Jørgensen, F. and 1313 Knudsen, K.L. (1994) A portable system for the measurement of sediment OSL in the 1314 field. Radiation Measurements, 23, 529-532.

1316 Porat, N., Lopez, G.I., Lensky, N., Elinson, R., Avni, Y., Elgart-Sharon, Y., Faershtein, 1317 G. and Gadot, Y. (2019) Using portable OSL reader to obtain a time scale for soil 1318 accumulation and erosion in archaeological terraces, the Judean Highlands, Israel. 1319 Quaternary Geochronology, 49, 65-70.

1321 Portenga, E.W. and Bishop, P. (2016) Confirming geomorphological interpretations 1322 based on portable OSL reader data. Earth Surface Processes and Landforms, 41, 4271323432.

1325 Portenga, E.W., Bishop, P., Gore, D.B. and Westaway, K.E. (2016) Landscape 1326 preservation under post-European settlement alluvium in the south-eastern Australian 1327 tablelands, inferred from portable OSL reader data. Earth Surface Processes and 1328 Landforms, 41, 1697-1707. 
1330 Preston, J., Sanderson, D., Kinnaird, T., Newton, A., Nitter, M., Coolen, J., Mehler, N. and

1331 Dugmore, A. (2020) Dynamic beach response to changing storminess of Unst, Shetland:

1332 implications for landing places exploited by Norse communities. Journal of Island and

1333 Coastal Archaeology, 15, 153-178. (https://doi.org/10.1080/15564894.2018.1555193).

1334

1335 Rhodes, E.J. (2011) Luminescence dating of sediment over the past 200,000 years.

1336 Annual Reviews in Earth and Planetary Sciences, 39, 461-488.

1337

1338 Rittenour, T. M. (2008) Luminescence dating of fluvial deposits: applications to

1339 geomorphic, palaeoseismic and archaeological research. Boreas, 37, 613-635.

1340

1341 Roberts, H.M., Durcan, J.A. and Duller, G.A.T. (2009) Exploring procedures for the

1342 rapid assessment of optically stimulated range finder ages. Radiation Measurements, $134344,582-587$.

1344

1345 Roberts, H.M., Duller, G.A.T., Gunn, M., Cousins, C.R., Cross, R.E. and Langstaff, D.

1346 (2018) Strategies for equivalent dose determination without heating, suitable for

1347 portable luminescence readers. Radiation Measurements, 120, 170-175.

1348

1349 Roberts H.M. and Wintle, A.G. (2001) Equivalent dose determinations for polymineralic

1350 fine-grains using the SAR protocol: application to a Holocene sequence of the Chinese

1351 Loess Plateau. Quaternary Science Reviews, 20, 859-864. 
1353 Rother, H., Lorenz, S., Börner, A., Kenzler, M., Siermann, N., Fülling, A., Hrynowiecka, 1354 A., Forler, D., Kuznetsov, V., Maksimov, F. and Starikova, A. (2019) The terrestrial 1355 Eemian to late Weichselian sediment record at Beckentin (NE-Germany): First results 1356 from lithostratigraphic, palynological and geochronological analyses. Quaternary 1357 International, 501, 90-108.

1359 Sanderson, D. C. W. and Kinnaird, T. C. (2019) Optically stimulated luminescence dating 1360 as a geochronological tool for late Quaternary sediments in the Red Sea region. In: Rasul, 1361 N. M. A. and Stewart, I. C. F., (Eds.) Geological Setting, Palaeoenvironment and 1362 Archaeology of the Red Sea: Springer International Publishing, pp. 685-707. ISBN 13639783319994079 (doi:10.1007/978-3-319-99408-6_31).

1365 Sanderson, D.C.W. and Murphy, S. (2010) Using simple portable measurements and 1366 laboratory characterisation to help understand complex and heterogenous sediment 1367 sequences for luminescence dating. Quaternary Geochronology, 5, 299-305.

1369 Sanderson, D.C.W., Bishop, P., Huston, I. and Boonsener, M. (2001) Luminescence 1370 characterization of quartz-rich cover sands from NE Thailand. Quaternary Science 1371 Reviews, 20, 893-900. 
1373 Sanderson, D.C.W., Bishop, P., Stark, M.T. and Spencer, J.Q. (2003) Luminescence 1374 dating of anthropologically reset canal sediments from Angkor Borei, Mekong Delta, 1375 Cambodia. Quaternary Science Reviews, 22, 1111-1121.

1376

1377 Sanderson D.C.W and Clark R.J., 1994, Pulsed Photostimulated Luminescence of Alkali 1378 Feldspars, Radiation Measurements 23, 633-639

1379

1380 Short, M.A. and Huntley, D.J. (1992) Infrared stimulation of quartz. Ancient TL, 10, 19138121.

1382

1383 Singhvi, A.K. and Porat, N. (2008) Impact of luminescence dating on geomorphological 1384 and paleoclimate research in drylands. Boreas, 37, 536-558.

1386 Smetana, F., Hayek, M., Bergmann, M., Brusl, H., Fugger, M., Gratzl, W., Kitz, E. and 1387 Vana, N. (2008) A portable multi-purpose OSL reader for UV dosimetry at workplaces. 1388 Radiation Measurements, 43, 516-519.

1390 Spooner, N.A. and Questiaux, D.G. (1989) Optical dating - Achenheim beyond the 1391 Eemian using green and infra-red stimulation. Long and short range limits in 1392 luminescence dating. Occasional Publication Vol 9. The Research Laboratory for 1393 Archaeology and the History of Art, Oxford. 
1395 Stang, D.M., Rhodes, E.J. and Heimsath, A.M. (2012) Assessing soil mixing processes 1396 and rates using a portable OSL-IRSL reader; preliminary determinations. Quaternary 1397 Geochronology, 10, 314-319.

1398

1399 Stone, A.E.C., Bateman, M.D. and Thomas, D.S.G. (2015) Rapid age assessment in 1400 the Namib Sand Sea using a portable luminescence reader. Quaternary 1401 Geochronology, 30, 134-140.

1402

1403 Stone, A., Bateman, M.D., Burrough, S.L., Garzanti, E., Limonta, M., Radeff, G. and 1404 Telfer, M.W. (2019) Using a portable luminescence reader for rapid age assessment of 1405 aeolian sediment for reconstructing dunefield landscape evolution in southern Africa. 1406 Quaternary Geochronology, 49, 57-64.

1407

1408 Takeuchi, T., Shibutani, T. and Hashimoto, T. (2008) Construction of a portable mini 1409 luminescence measurement system equipped with a miniature X-ray generator.

1410 Geochronometria, 30, 17-22.

1411

1412 Telfer, M.W. (2011) Growth by extension and reworking of a southwestern Kalahari

1413 linear dune. Earth Surface Processes and Landforms, 36, 1125-1135.

1415 Thomsen, K.J., Jain, M., Murray, A.S., Denby, P.M., Roy, N. and Bøtter-Jensen, L. (2008)

1416 Minimizing feldspar OSL contamination in quartz UV-OSL using pulsed blue stimulation. 1417 Radiation Measurements, 43, 752-757. 
1419 Vervust, S., Kinnaird, T.C., Herring, P. and Turner, S. (2020) Dating earthworks using 1420 optically-stimulated luminescence profiling and dating (OSL-PD): the creation and 1421 development of prehistoric field boundaries at Bosigran, Cornwall (UK). Antiquity, 94, $1422 \quad 420-436$.

1423

1424 Wallinga, J., Murray, A.S. and Bøtter-Jensen, L. (2002) Measurement of the dose in 1425 quartz in the presence of feldspar contamination. Radiation Protection Dosimetry, 101, $1426 \quad 367-370$.

1428 Wintle, A.G. (2008) Luminescence dating: where it has been and where it is going.

1429 Boreas, 37, 471-482.

1430

1431

1432 ACKNOWLEDGEMENTS

1433 Susan Fryters is thanked for proofreading several versions of this manuscript. 


\section{FIGURE CAPTIONS}

1442

1443 Figure 1 (a) The third-generation SUERC portable luminescence reader. (b) The 1444 instrument weighs less than $5 \mathrm{~kg}$ and can be transported in a briefcase-sized holder.

1445

1446 Figure 2 Typical luminescence signal curves obtained using the SUERC portable OSL 1447 reader. (a) IRSL and post-IR blue OSL shine-down curves obtained following stimulation 1448 of a bulk sample of aeolian sands from central Alberta for 120 seconds. (b) Same sample 1449 stimulated for 120 seconds using a pulsed signal (15 $\mu$ s up and $15 \mu$ down). In both 1450 cases, dark count periods of $10 \mathrm{~s}$ before and after stimulation were used.

1451

1452 Figure 3 Illustration of IRSL and blue OSL signals measured using the portable OSL 1453 reader that increase gradually with depth (and age) in a depositional sequence. (a) IRSL 1454 and blue OSL signals measured on samples from an aeolian dune in central Alberta, 1455 Canada. (Adapted from Munyikwa et al., 2012). (b) signals from fluvial deposits (sand 1456 and silty clay) from Grabben Gullen Creek in SE Australia. (Adapted from Portenga et al., 1457 2016).

1458

1459 Figure 4 Luminescence signals from a coastal aeolian dune sequence in Norfolk County, 1460 UK deposited over a relatively short period of time. The entire profile accumulated over a 1461 ca. 15 year period. (Adapted from Bateman et al., 2015). 
1463 Figure 5 IRSL and post-IR blue OSL profiles showing inverted signal distribution. (a) 1464 Signals from a Neolithilic ditch exposed in a quarry section at Cava Petrilli, Italy. The 1465 stratigraphy shows a darker lower ditch fill, possibly an agricultural soil overlain by an 1466 upper fill comprising post-abandonment backfill derived from poorly bleached former 1467 substrate material (Adapted from Sanderson and Murphy, 2010). (b) Signals from fluvial 1468 sediment from Grabben Gullen Creek, southeast Australia. Poorly bleached post1469 settlement alluvium (PSA) overlies swampy meadow (SM) alluvium that was well 1470 bleached at deposition (Adapted from Muñoz-Salinas et al., 2014).

1472 Figure 6 IRSL and OSL profiles depicting an interface between units of different age. (a) 1473 Signals from an abandoned fluvial channel in Cambodia. Units I/II were radiocarbon dated 1474 as modern while organic material from III/IV returned an age of ca. $6 \mathrm{ka}$ (Adapted from 1475 Muñoz-Salinas et al., 2011). (b) A profile and signals from aeolian sands (unit 6) aged 1476 about 14 ka overlying last Interglacial lake sediment (ca 114-118 ka) from Beckentin, NE 1477 Germany (Adapted from Rother et al., 2019).

1479 Figure 7 The variation of IRSL and post-IR signal intensities with distance from the ice 1480 margin recorded from braid-bar and side-attached bar deposits at Bergsetdalen, 1481 Jostedalen, southern Norway. Overall, signals are higher and the scatter in signals 1482 greater closer to source than further downstream (Adapted from King et al., 2014).

1484 Figure 8 The variation of IRSL 'effective ages' with depth for three different monoliths 1485 sampled in the San Gabriel Mountains, California. Note that the 'effective ages' do not 
1486 equal depositional ages of the sediments since the ages were obtained from mixed grain 1487 populations. (a) For Monolith A, two episodes during which the sediment may have been 1488 at the surface are shown. (b) For Monolith B, the high degree of fluctuation in 'effective 1489 age' was thought to indicate a high soil turnover history, possibly resulting from extensive 1490 bioturbation. (c) For Monolith C, the constant signals in the upper part of the soil profile 1491 suggested the presence of a soil macropore that was filled by surface sediment during a 1492 storm or slumping event (Adapted from Stang et al., 2012).

Figure 9 Variation in IRSL signals with distance downstream along ravines in (a) areas under conservation and (b) areas under natural vegetation. In areas under conservation, 1496 signals increase downstream because the stream entrains poorly bleached samples with 1497 distance. In areas under natural vegetation, sediment is only sourced upstream and it 1498 bleaches with distance (Adapted from Munoz-Salinas et al., 2018).

1500 Figure 10 SGC constructed by Munyikwa and Brown (2014) for central Alberta using 1501 portable OSL reader measurements. Samples used to construct the curve were collected 1502 from six different sites. To approximate $D_{e}$ for a sample of unknown age, a sample's 1503 natural signal is measured using the portable OSL reader after which a test dose is 1504 applied (gamma ray) to normalize the natural signal. Interpolating the normalized signal 1505 into SGC provides the required $D_{e}$ value (Adapted from Munyikwa and Brown, 2014). 
1507 Figure 11 Regression models of southern African aeolian dune sample OSL ages

1508 plotted against portable OSL reader bulk post-IR blue OSL signals. Four main regional

1509 curves were obtained (Adapted from Stone et al., 2019).

1510

1511 Figure 12 (a) A plot of luminescence signal versus depth in a pit excavated in an 1512 agricultural terrace in the Judean Highlands of Israel . Also shown are sampling positions

1513 of samples dated using standard OSL protocols. (b) A regression model of sample age

1514 versus blue OSL signal. The derived function was then used to calculate ages of samples

1515 of unknown age whose signals are indicated in Figure 12(a) (Adapted from Porat et al., 1516 2019).

1517

1518 Figure 13 Quadratic curve derived from portable OSL reader signals plotted against

1519 ages established using radiocarbon and standard OSL procedures. Data shown above

1520 are after sieving and removal of magnetic minerals using hand magnet (Adapted from

1521 Gray et al., 2018).

1522

1523

1524

1525

1526

1527

1528

1529 
TABLE 1 Summary of major positive features and drawbacks of portable OSL readers examined in this paper

Portable OSL Reader Major Positive Attributes
developed by:

\begin{tabular}{|c|c|c|}
\hline Poolton et al. (1994) & $\begin{array}{ll}\text { - } & \text { Lightweight }(<5 \mathrm{~kg}) \text {. } \\
\text { - } & \text { Sample cartridge can hold up to } 12 \\
\text { - } & \text { Samples. } \\
\text { - } & \text { normalisation. } \\
\text { - } \quad \text { Dedicated sample bleaching lamp (blue). } & \text { CW sample stimulation mode }\end{array}$ & $\begin{array}{ll}\text { - } & \text { Primarily targets feldspar for } \\
\text { analysis (source for quartz } \\
\text { stimulations can be added). } \\
\text { - } \quad \text { No pulsed stimulation. } \\
\text { - } \quad \text { No irradiation source. } \\
\text { - } \text { Instrument never developed } \\
\text { - } \quad \text { past prototype version. } \\
\text { Requires 12V DC power } \\
\text { source. }\end{array}$ \\
\hline Takeuchi et al. (2008) & $\begin{array}{ll}\text { - } & \text { Sample cartridge can hold up to } 13 \\
\text { - } & \text { } \\
\text { - } & \text { HTL and } \\
\text { - } & \text { fes both IR stimulation source (for } \\
\text { - } & \text { Performs both CW and pulsed-OSL } \\
\text { sample stimulation (can gate photon } \\
\text { counting during pulsed-OSL). } \\
\text { - } \quad \text { Sample heating possible (up to } 600^{\circ} \mathrm{C} \text { ) } \\
\text { - } \quad \text {-ray generator for artificial irradiation. } \\
\text { Can operate on } 5 V \text { DC power source } \\
\text { (when heating not required). }\end{array}$ & $\begin{array}{ll}\text { - } & \text { Weighs about } 15 \mathrm{~kg} \\
\text { - } \quad \text { Instrument never developed } \\
\text { past prototype version. }\end{array}$ \\
\hline
\end{tabular}

\begin{tabular}{lll}
\hline SUERC (Sanderson and & - & Lightweight $(<5 \mathrm{~kg})$. \\
Murphy, 2010) & - Has both IR stimulation source (for \\
& feldspar) and blue light (for quartz). \\
- & Simple and robust operation. \\
- & Instrument used in multiple studies in a \\
- & wide range of environments. \\
Performs CW and pulsed-OSL sample & stimulation. \\
- & Can operate on $5 \mathrm{~V}$ DC power source.
\end{tabular}

- No irradiation source.

- Sample tray only holds one sample at a time.

- No heating element

- Cannot gate photon counting during pulsed-OSL.

\footnotetext{
Kook et al. (2011) • $\quad$ Lightweight (about $5 \mathrm{~kg}$ ).

- X-ray generator for artificial irradiation.

- $\quad$ Performs both CW and pulsed-OSL sample stimulation (can gate photon counting during pulsed-OSL).

- Has IR and blue LED sources for sample bleaching.

- $\quad$ Sampler doubles as sample holder during measurement (prevents sample exposure to light when sampling).

- $\quad$ Sample heating possible (up to $>250^{\circ} \mathrm{C}$ )

- $\quad$ Can operate on 9V DC power source.

- $\quad$ Primarily targets quartz for analysis (no IRSL sources for measurement.)

- Power requirement up to $45 \mathrm{~V}$ DC when heating required.

- Samples with organic material may be difficult to heat.
} 
TABLE 2 Studies that have conducted luminescence profiling using a portable luminescence reader

\begin{tabular}{ll}
\hline Environment & References and Study Region \\
\hline Fluvial I & Muñoz-Salinas et al., 2011 (Cambodia); \\
Colluvial & Kinnaird et al., 2013 (SW Cyprus); Muñoz- \\
& Salinas et al., 2014 (SE Australia); \\
& Palamakumbura et al., 2016 (N Cyprus); \\
& Portenga and Bishop, 2016 (SE Australia); \\
& Portenga et al., 2016 (SE Australia); Kappler, \\
& et al, 2018 (NE Germany); Lichtenberger et \\
& al., 2019 (Jordan).
\end{tabular}

\section{Application}

OSL profiling used to identify boundaries between units of different age (unconformities) or units with different signals resulting from different mineralogies (e.g. quartz to feldspar ratio). Different signals may also be an artifact of provenance. Signals may provide information on transport mechanisms. Enables the screening of sediment suitable for OSL dating.

Coastal Sanderson and Murphy, 2010 (Thailand);
Kinnaird et al., 2012 (Orkney, UK); Bateman et al., 2015 (Norfolk, UK); Kinnaird et al., 2015 (Shetland Islands, UK); Brill et al., 2017 (NW Australia); Preston et al., 2020 (Shetland, UK).

OSL profiling used to identify interfaces between units of different age or between wellbleached and poorly bleached sediments (e.g. Tsunami-wave deposits atop onshore deposits). Luminescence signals provide information on possible transport mechanisms. Enables the selection of sediment appropriate for dating.

\begin{tabular}{ll}
\hline Archaeological & Sanderson and Murphy, 2010 (Italy); Ghilard \\
& et al., 2015 (Avignon, France); Kinnaird et al. \\
& 2017 (Catalonia, Spain); Porat et al., 2019 \\
& (Israel); Lichtenberger et al., 2019 (Jordan); \\
& Vervust, et al., 2019 (Cornwall, UK).
\end{tabular}

Examined features include ditch fills,
earthworks, historical bridge construction, mine workings and agricultural terraces. Profiling used to identify depositional units of different age and to distinguish priority targets for dating using OSL methods.

\begin{tabular}{ll}
\hline Aeolian (inland) & Munyikwa et al., 2012 (AB, Canada); Rother \\
& et al., 2019 (NW Germany)
\end{tabular}

OSL profiling used to differentiate between units that were bleached well prior to burial and those that were not. Profiling also used to identify interfaces between depositional units of different age (unconformities).
Glaciolacustrine Píšková et al., 2019 (Vega Island, Antarctic Peninsula)

OSL profiling conducted on lake sediment cores to identify samples for dating using regular OSL methods.

\section{Offshore/marine Sanderson and Kinnaird, 2019 (Red Sea)}

OSL profiling of marine cores allowed the identification Pleistocene units, beneath more recent Holocene sediments on the continental shelf of the Red Sea near the Farasan Islands

\begin{tabular}{lllll}
\hline Paraglacial & $\begin{array}{l}\text { Muñoz-Salinas } \\
\text { Gredos, Spain) }\end{array}$ & et al., 2013 & $\begin{array}{l}\text { (Sierra de } \\
\end{array}$ & $\begin{array}{l}\text { Profiling of debris flows and fluvial deposits in } \\
\text { paraglacial landscapes to obtain relative } \\
\text { chronology of deposition prior to sampling for } \\
\text { OSL dating. }\end{array}$
\end{tabular}


1546 TABLE 3 Studies that have employed portable OSL readers to explore sediment bleaching

1547

\begin{tabular}{lll}
\hline Environment & $\begin{array}{l}\text { References and Study } \\
\text { Region }\end{array}$ & Applications \\
\hline Pedogenic / soils & $\begin{array}{l}\text { Stang et al., 2012 } \\
\text { (California, USA) }\end{array}$ & $\begin{array}{l}\text { OSL reader signals used to study patterns and rates of soil } \\
\text { mixing. Results suggest that there are multiple processes that are } \\
\text { operating to result in vertical movement of mineral grains in soils, } \\
\text { including bioturbation and filling of macropore by sediment during } \\
\text { storm or slump events. Findings allow soil turnover history to be } \\
\text { calculated. }\end{array}$ \\
& &
\end{tabular}

$\begin{array}{ll}\text { Glaciofluvial } & \begin{array}{l}\text { King et al., } 2014 \\ \text { (Jostdalen, Norway) }\end{array}\end{array}$

Modern glacial sediments analyzed in an effort to gain insight into catchment-scale sediment bleaching processes. Results show that high-magnitude processes of low frequency are characterized by poor sediment bleaching whereas lowmagnitude events that occur with higher frequency are associated with best chances for sediment bleaching.

\begin{tabular}{lll}
\hline $\begin{array}{l}\text { Orogenic } \\
\text { Itectonic uplift }\end{array}$ & $\begin{array}{l}\text { Castillo et al., 2014 } \\
\text { (Western Mexico) }\end{array}$ & $\begin{array}{l}\text { Portable OSL reader measurements used to investigate rates of } \\
\text { erosion in the Jalisco Block in order to assess geomorphic }\end{array}$ \\
& $\begin{array}{l}\text { response to differential uplift. Results showed that higher uplift } \\
\text { rates in the northern sector produces higher relief that }\end{array}$ \\
& $\begin{array}{l}\text { corresponds with more turbid flows such that sediments in } \\
\text { riverbeds are poorly bleached. }\end{array}$
\end{tabular}

\begin{tabular}{ll}
\hline Volcanic /coastal & Muñoz-Salinas et al., \\
I fluvial & $2017 a$ (Mexico)
\end{tabular}

Portable OSL reader used to examine young sediments $(<2 \mathrm{ka})$ from volcanic, coastal and fluvial environments in order to differentiate between sediments that are most effectively zeroed from those that are poorly bleached. Findings indicate that debris flow sediments are most likely to be poorly bleached. IRSL signals of volcanic ash, lavas, and sand beach and dune deposits show minimal scatter whereas blue OSL signals display significant scatter, likely due to charge transfer or low quartz sensitivity.

\begin{tabular}{ll}
\hline Fluvial & Muñoz-Salinas et al., \\
& 2017b (Oaxaca, Mexico); \\
& Muñoz-Salinas and \\
& Castillo, 2018 (Izta-Popo, \\
& Mexico)
\end{tabular}

Residual IRSL and blue OSL signals in fluvial sediments were investigated using a portable OSL reader to gain insight into mechanisms involved during debris flows. Results suggest sediment grains are transported without stratification; deposits from steeper channels have higher signals as a result of more turbid flows. Another study compared degree of bleaching in sediment in natural areas to that in areas under conservation. Results show that sediment in ravines that cut into natural forest and alpine grassland are better bleached than sediment in alpine grassland under conservation practice. Hence, curbing the effects of human activity in the area appears to be ineffective.

Lichtenberger et al., 2019 (Wadi Suf, Jordan)

Portable OSL reader used to examine sediment in three sections along Wadi Suf in a study looking at the relationship between urban settlement and riverine systems in semi-arid environments. Results show that both IRSL and blue OSL signal intensities increased downstream suggesting increase in sensitivity due to repeated erosion-deposition cycles. 
TABLE 4 Select studies that have employed portable OSL readers to approximate sample age

\begin{tabular}{lll}
\hline Environment & $\begin{array}{l}\text { References and } \\
\text { Study Region }\end{array}$ & Methodology \\
\hline $\begin{array}{l}\text { Aeolian } \\
\text { (inland) }\end{array}$ & $\begin{array}{l}\text { Munyikwa and Brown, } \\
2014 \text { (AB, Canada) }\end{array}$ & $\begin{array}{l}\text { SGC was constructed using regeneration method (Figure 6); signals } \\
\text { were normalized using a gamma source }\left({ }^{137} \mathrm{Cs}\right) \text {. SGC showed linear } \\
\text { growth up to at least 100 Gy. } \mathrm{D}_{\mathrm{e}} \mathrm{S} \text { of natural samples of unknown ages }\end{array}$ \\
& $\begin{array}{l}\text { were determined by acquiring a portable OSL reader signal of bulk } \\
\text { sample and normalizing it and then comparing with SGC for } \\
\text { corresponding De. Large uncertainties but results generally show } \\
\text { capacity to approximate } \mathrm{D}_{\mathrm{e}} \text { for samples within region. }\end{array}$
\end{tabular}

\begin{tabular}{lll}
\hline $\begin{array}{l}\text { Aeolian } \\
\text { (inland) }\end{array}$ & $\begin{array}{l}\text { Stone et al., 2015 } \\
\text { (Namib Sand Sea, }\end{array}$ & $\begin{array}{l}\text { Study aimed to convert portable OSL readings into luminescence age } \\
\text { estimates. Samples whose ages have been determined using standard } \\
\text { Namibia) }\end{array}$ \\
& $\begin{array}{l}\text { luminescence dating protocols were measured using a portable OSL } \\
\text { reader. Acquired signals were plotted against sample age to produce a } \\
\text { regional calibration curve (linear regression) for rapid age } \\
\text { approximation. Resulting calibration curve tested by measuring } \\
\text { portable signals for samples whose ages are known but were not used } \\
\text { to construct the curve. Predicted sample ages consistent with the real } \\
\text { ages. }\end{array}$
\end{tabular}

Coastal Brill et al., 2016 (Phra, Thong, Thailand)

$\begin{array}{ll}\text { Coastal } & \text { Brill et al., } 2016 \text { (Phra, } \\ \text { Thong, Thailand) }\end{array}$

Study conducted using both luminescence profiling and SGCs of samples whose ages had previously been dated using standard luminescence dating protocols. SGC constructed using bulk samples and a regular Risø TL-DA system. The broad depositional chronology ranging from late Holocene to last interglacial can be elucidated by the SGC data. However, the ages have large uncertainties, making it difficult to identify shorter events.
Gray et al., 2018 (Las Vegas Valley, Nevada, USA)
Study analyzed the relationship between portable OSL reader signals of paleowetland deposits of middle to late Pleistocene age and their absolute ages that had been determined using standard luminescence dating protocols to see if portable OSL reader signals can reliably be used to predict sample ages at the site. Sediment sources are thought to be aeolian and fluvial. Plots of sample age versus IRSL/OSL signals fitted with a quadratic equation. Despite significant scatter, results indicate that portable OSL reader signals correlate with sample age.

Study builds on work by Stone et al. (2015) by using a larger set of samples. Portable OSL reader signals of 144 aeolian samples from four regions in southern Africa whose ages have been established using regular luminescence dating protocols were acquired. A regression analysis of the signals versus established age of samples shows that there are region-specific relationships between sample age and signals that can be used to predict approximate ages of samples of unknown age. Overall, the data suggest that the large number of samples used in the regression curve improve the quality of the results. Results were tested statistically using coefficient of determination and root mean square error. Findings indicate that there is a strong correlation between portable OSL reader signals and sample age, and regression curves can be used to predict ages reliably.

A series of portable OSL sequences $(>58)$ were expressed as apparent ages by cross calibration to full OSL dating samples. Bayesian sequences of the interdigitated chronologies of both the OSL dates, and the larger number of profiling apparent ages were developed and used to generalise the chronostratigraphy of the site. 

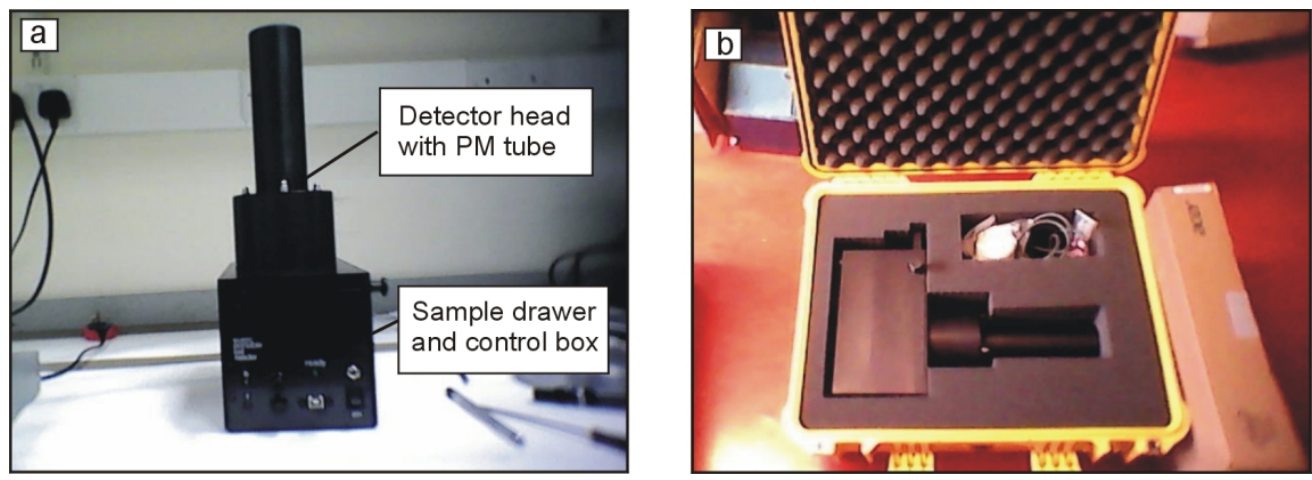

Figure 1 (a) The third-generation SUERC portable luminescence reader. (b) The instrument weighs less than $5 \mathrm{~kg}$ and can be transported in a briefcase-sized holder. 

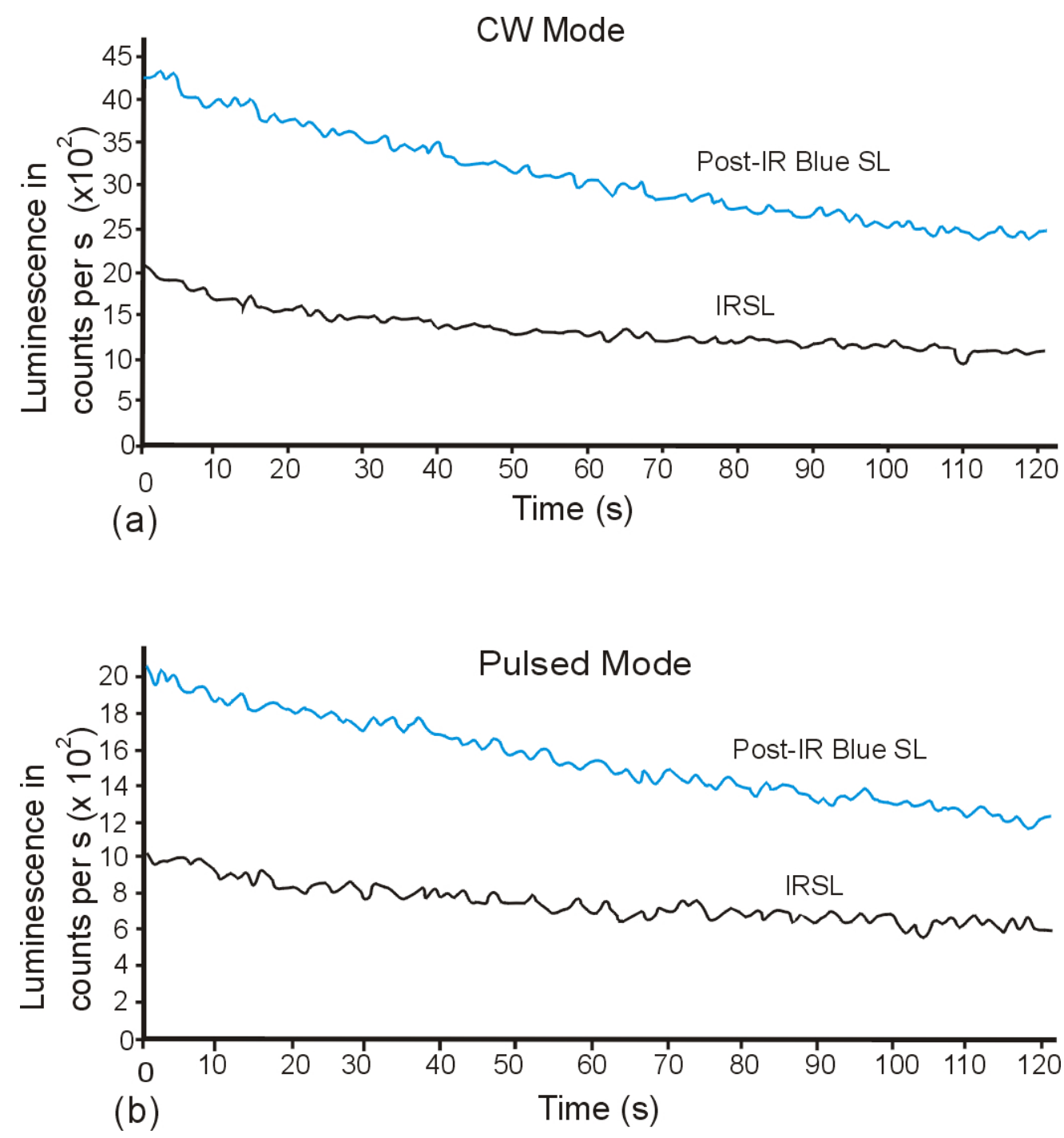

Figure 2 Typical luminescence signal curves obtained using the SUERC portable OSL reader. (a) IRSL and post-IR blue OSL shine-down curves obtained following stimulation of a bulk sample of aeolian sands from central Alberta for 120 seconds. (b) Same sample stimulated for 120 seconds using a pulsed signal (15 $\mu$ s up and $15 \mu \mathrm{s}$ down). In both cases, dark count periods of $10 \mathrm{~s}$ before and after stimulation were used. 

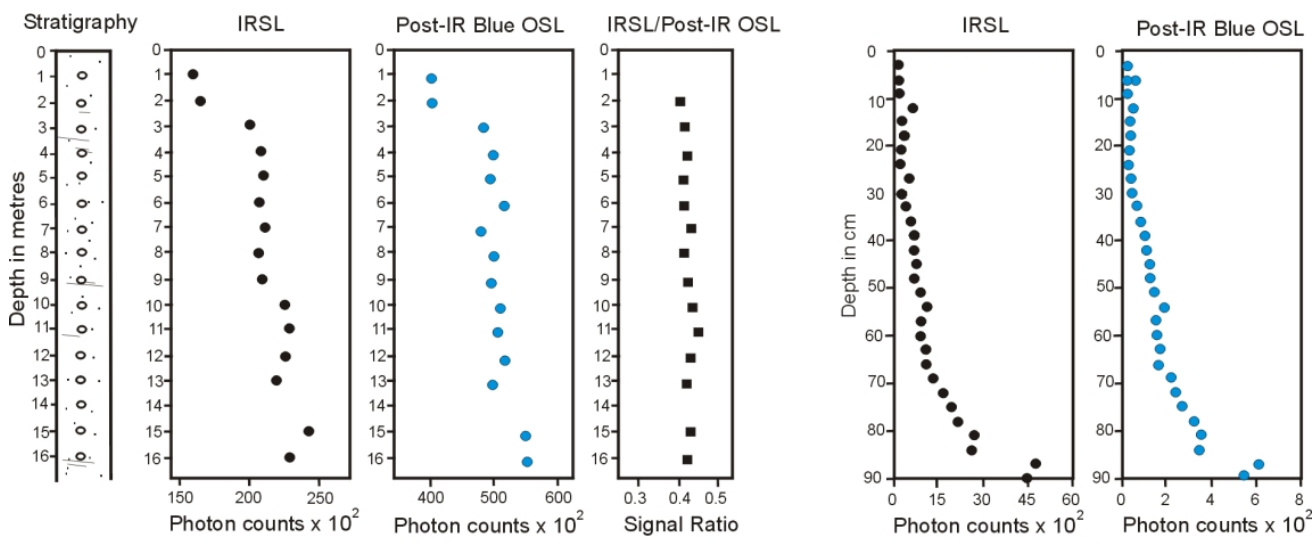

(a)

(b)

Eolian dune sand

- IRSL signal

- IRSL/Post-IR blue OSL ratio

- Portable OSL sample

- Post-IR blue OSL signal

Figure 3 Illustration of IRSL and blue OSL signals measured using the portable OSL reader that increase gradually with depth (and age) in a depositional sequence. (a) IRSL and blue OSL signals measured on samples from an aeolian dune in central Alberta, Canada (Adapted from Munyikwa et al., 2012). (b) signals from fluvial deposits (sand and silty clay) from Grabben Gullen Creek in SE Australia (Adapted from Portenga et al., 2016). 


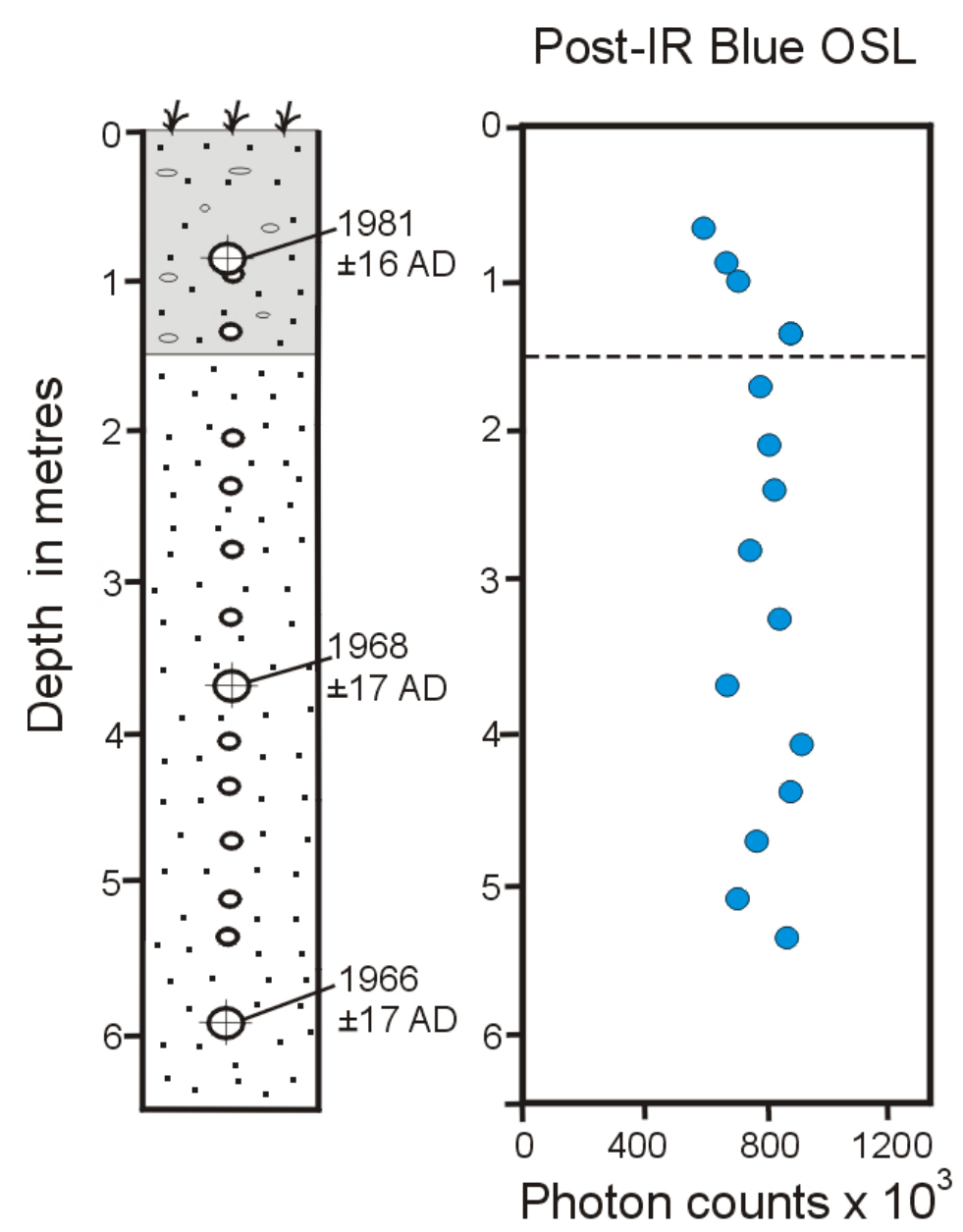

\section{$\because \because$ Sand Unit $1 \quad$ S Regular OSL dating sample $\because \because$ Sand Unit 2 - Post-IR OSL signal - Portable OSL sample}

Figure 4 Luminescence signals from a coastal aeolian dune sequence in Norfolk County, UK deposited over a relatively short period of time. The entire profile accumulated over a ca. 15 year period (Adapted from Bateman et al., 2015). 

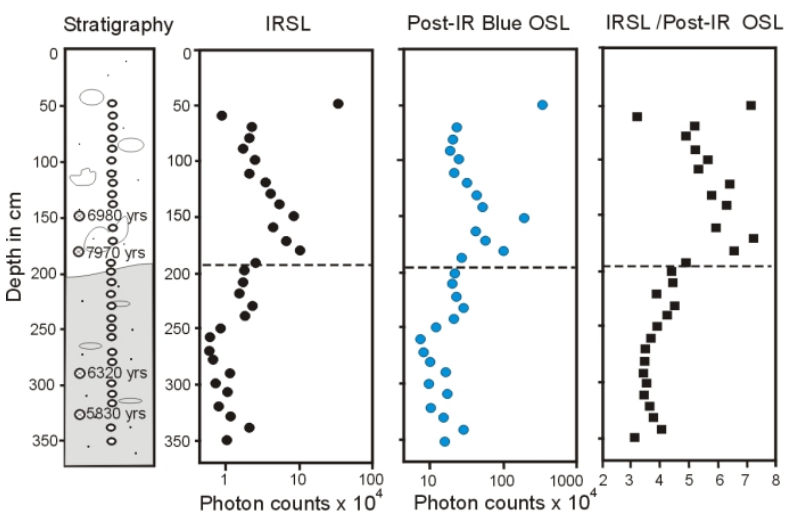

(a)
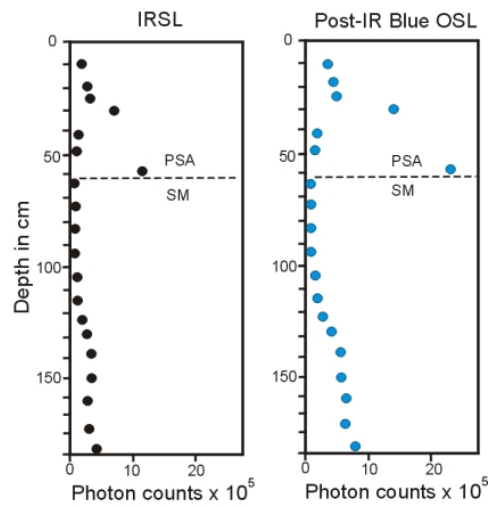

Upper ditch fill

$\circ$

Portable OSL sample

$\bullet$

Post-IR blue OSL signal

(b)

Lower ditch fill

- IRSL signal

- IRSL/Post IR Oblue OSL ratio

Figure 5 IRSL and post-IR blue OSL profiles showing inverted signal distribution. (a) Signals from a Neolithilic ditch exposed in a quarry section at Cava Petrilli, Italy. The stratigraphy shows a darker lower ditch fill, possibly an agricultural soil overlain by an upper fill comprising post-abandonment backfill derived from poorly bleached former substrate material (Adapted from Sanderson and Murphy, 2010). (b) Signals from fluvial sediment from Grabben Gullen Creek, southeast Australia. Poorly bleached post-settlement alluvium (PSA) overlies swampy meadow (SM) alluvium that was well bleached at deposition (Adapted from Muñoz-Salinas et al., 2014). 


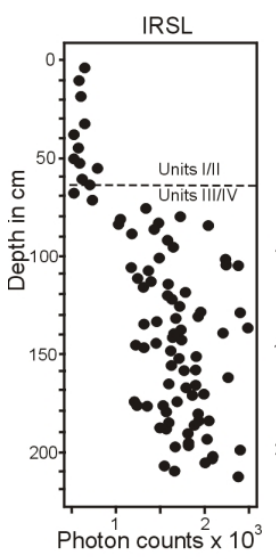

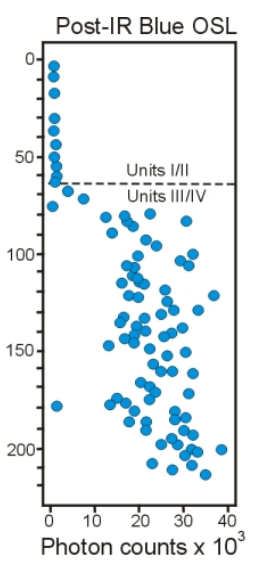

(a)
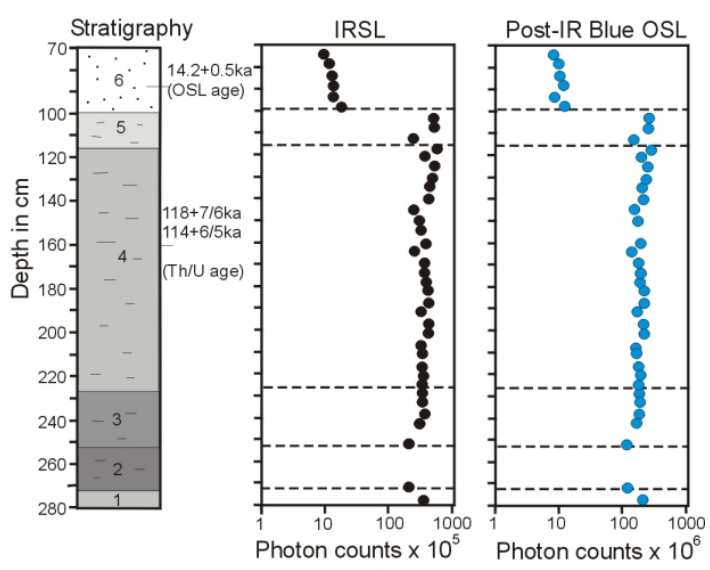

(b)

- IRSL

- Post-IR Blue OSL

Figure 6 IRSL and OSL profiles depicting an interface between units of different age. (a) Signals from an abandoned fluvial channel in Cambodia. Units I/II were radiocarbon dated as modern while organic material from III/IV returned an age of ca, 6 ka (Adapted from Muñoz-Salinas et al., 2011). (b) A profile and signals from aeolian sands (unit 6) aged about 14 ka overlying last Interglacial lake sediment (ca 114-118 ka) from Beckentin, NE Germany (Adapted from Rother et al., 2019). 


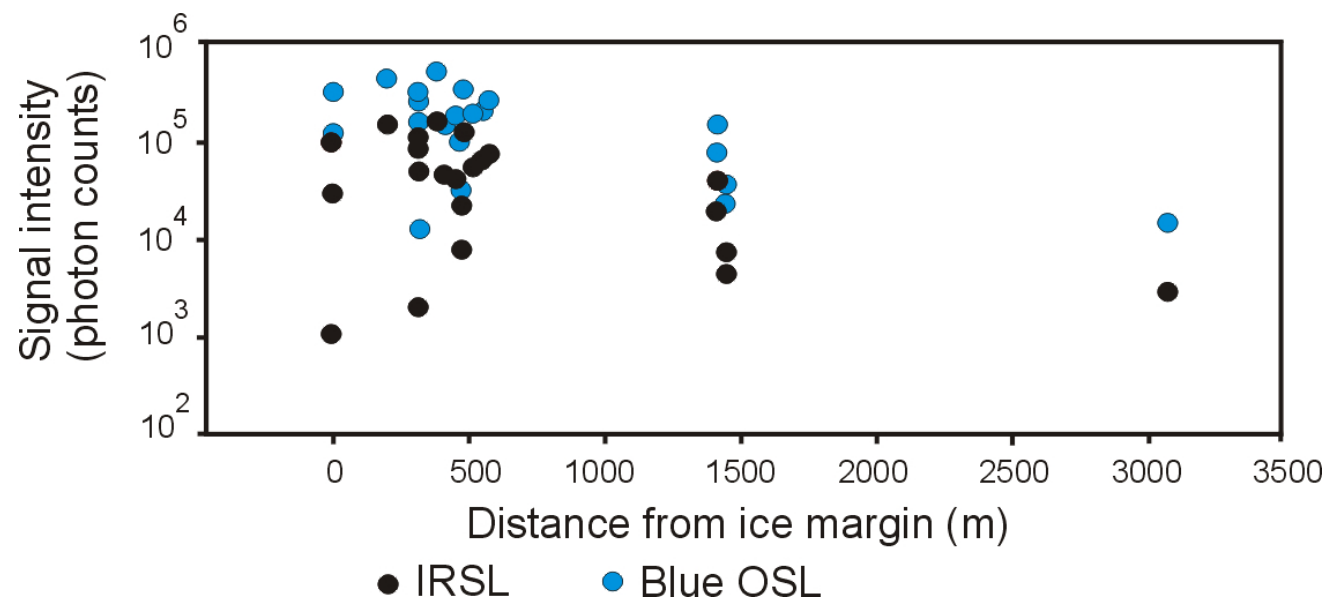

Figure 7 The variation of IRSL and post-IR signal intensities with distance from the ice margin recorded from braid-bar and side-attached bar deposits at Bergsetdalen, Jostedalen, southern Norway. Overall, signals are higher and the scatter in signals greater closer to source than further downstream (Adapted from King et al., 2014). 


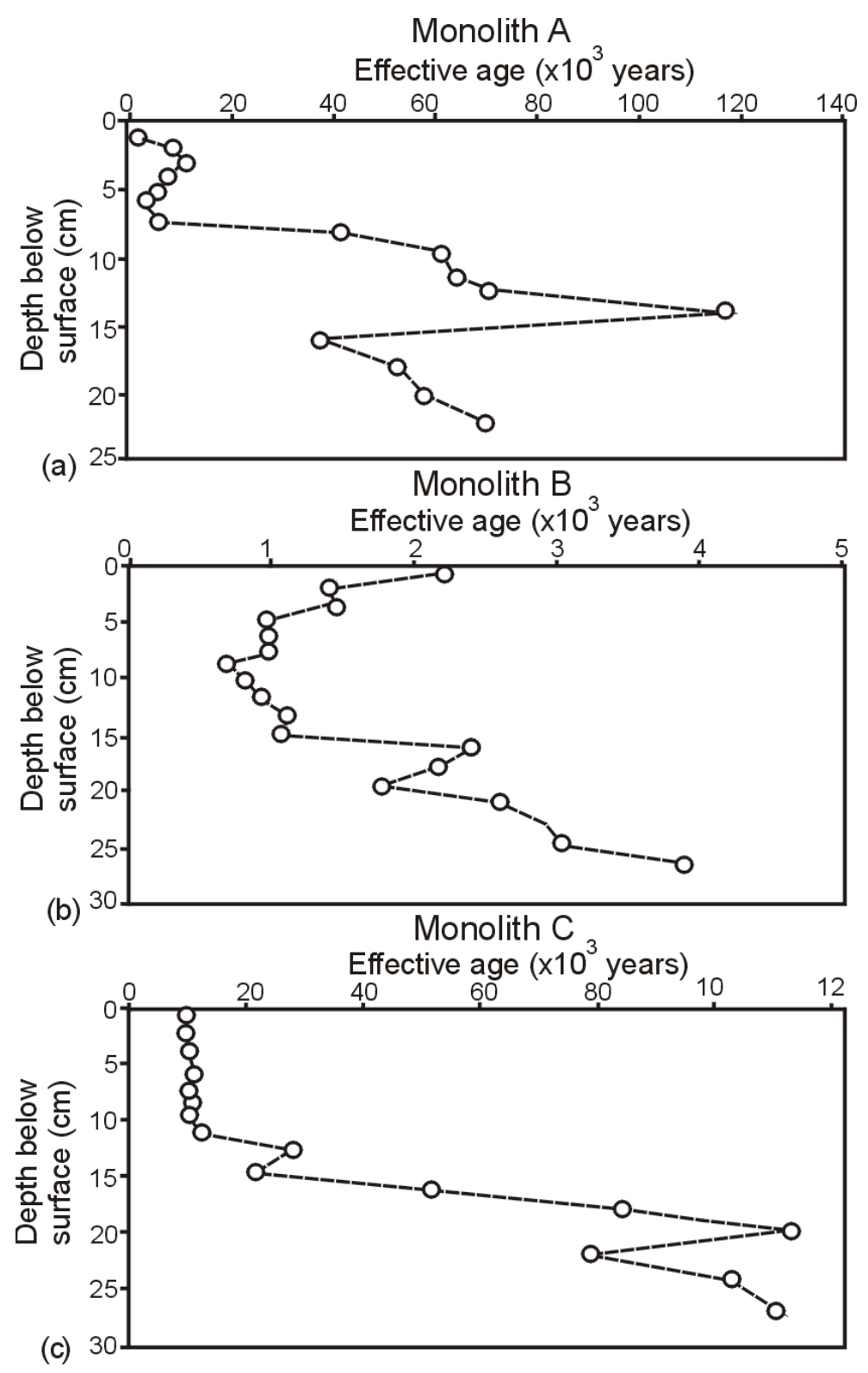

Figure 8 The variation of IRSL 'effective ages' with depth for three different monoliths sampled in the San Gabriel Mountains, California. Note that the 'effective ages' do not equal depositional ages of the sediments since the ages were obtained from mixed grain populations. (a) For Monolith A, two episodes during which the sediment may have been at the surface are shown. (b) For Monolith B, the high degree of fluctuation in

'effective age' was thought to indicate a high soil turnover history, possibly resulting from extensive

bioturbation. (c) For Monolith $\mathrm{C}$, the constant signals in the upper part of the soil profile suggested the presence of a soil macropore that was filled by surface sediment during a storm or slumping event (Adapted from Stang et al., 2012). 

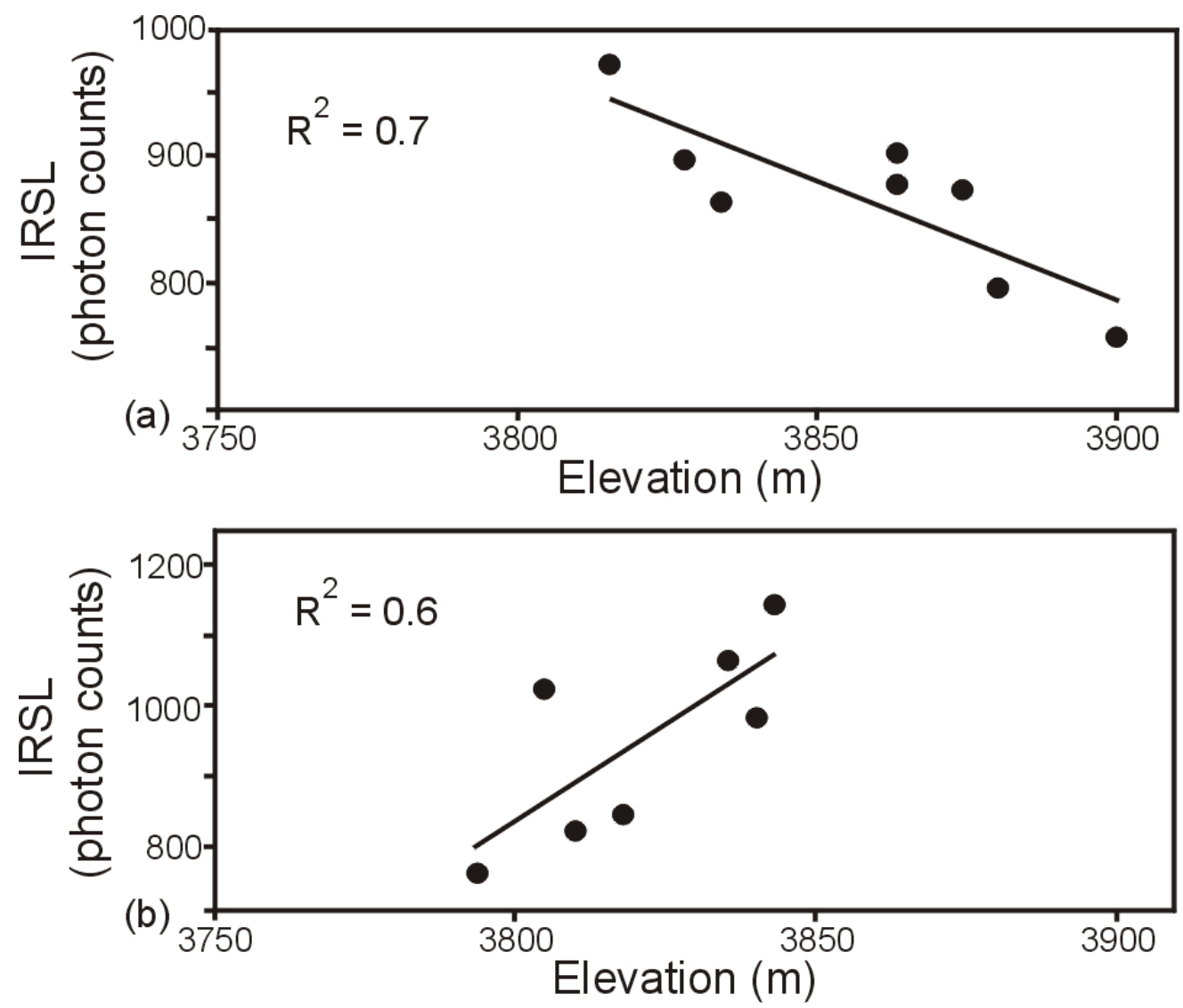

Figure 9 Variation in IRSL signals with distance downstream along ravines in (a) areas under conservation and (b) areas under natural vegetation. In areas under conservation, signals increase downstream because the stream entrains poorly bleached samples with distance. In areas under natural vegetation, sediment is only sourced upstream and it bleaches with distance (Adapted from Munoz-Salinas et al., 2018). 


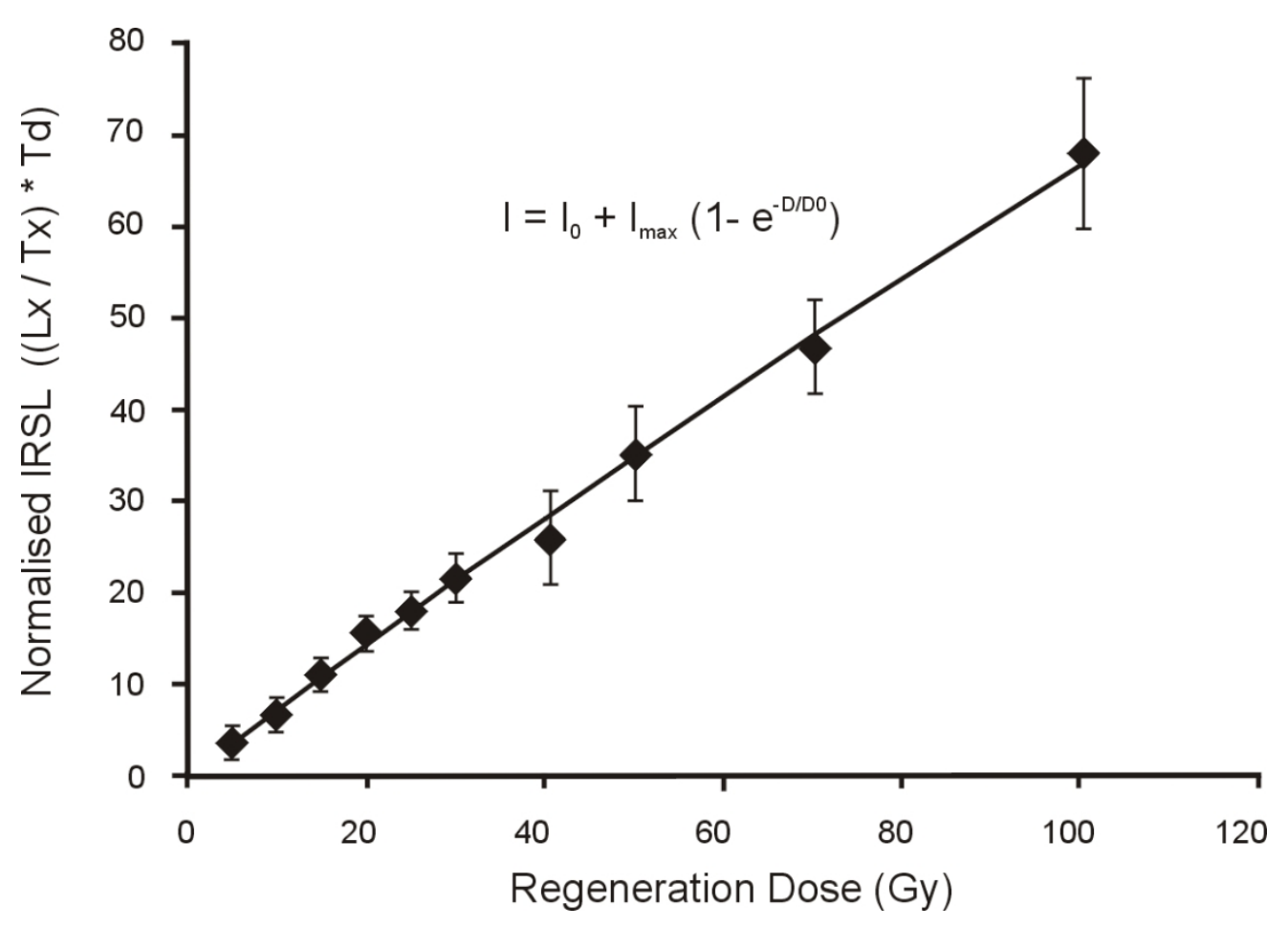

Figure 10 SGC constructed by Munyikwa and Brown (2014) for central Alberta using portable OSL reader measurements. Samples used to construct the curve were collected from six different sites. To approximate the equivalent dose (De) of a sample of unknown age, a sample's natural signal is measured using the portable OSL reader after which a test dose is applied (gamma ray) to normalize the natural signal. Interpolating the normalized signal into SGC provides the required De value (Adapted from Munyikwa and Brown, 2014). 


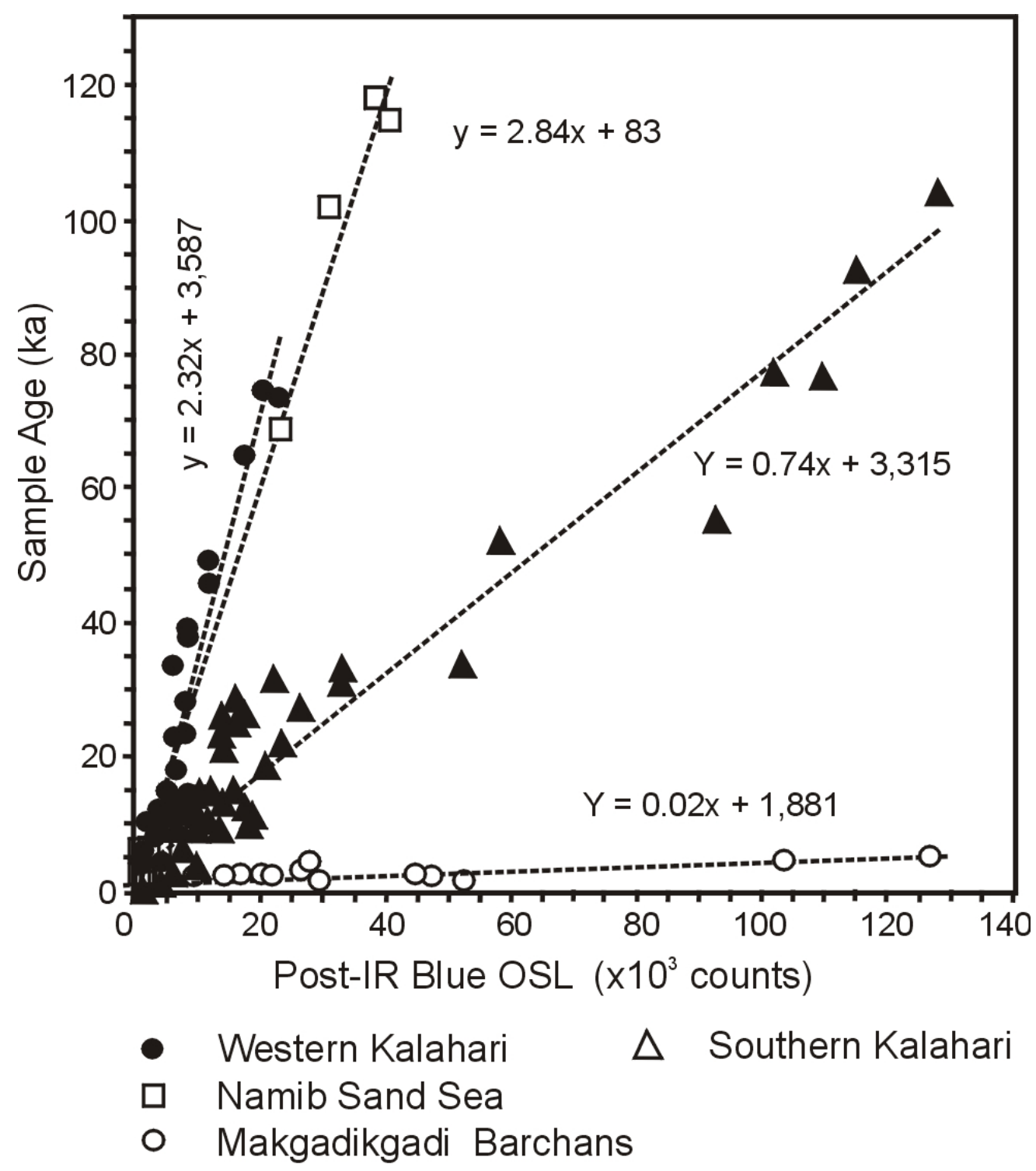

Figure 11 Regression models of southern African aeolian dune sample OSL ages plotted against portable OSL reader bulk post-IR blue OSL signals. Four main regional curves were obtained (Adapted from Stone et al., 2019). 


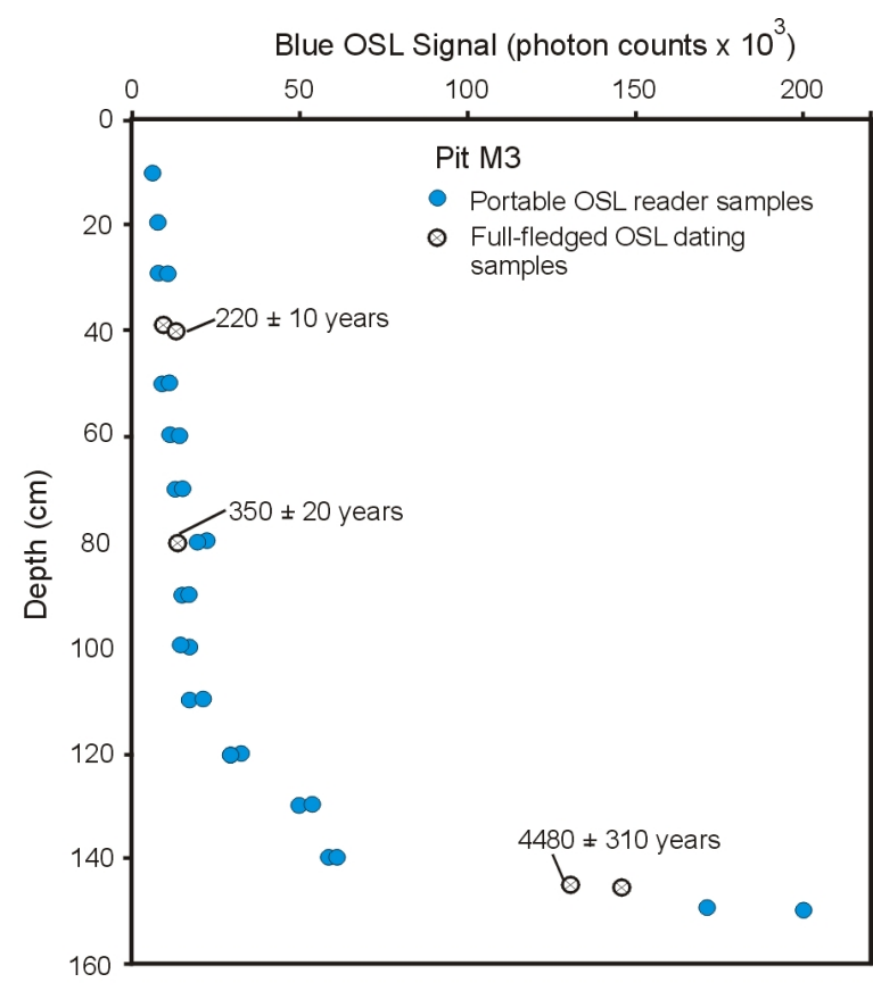

(a)

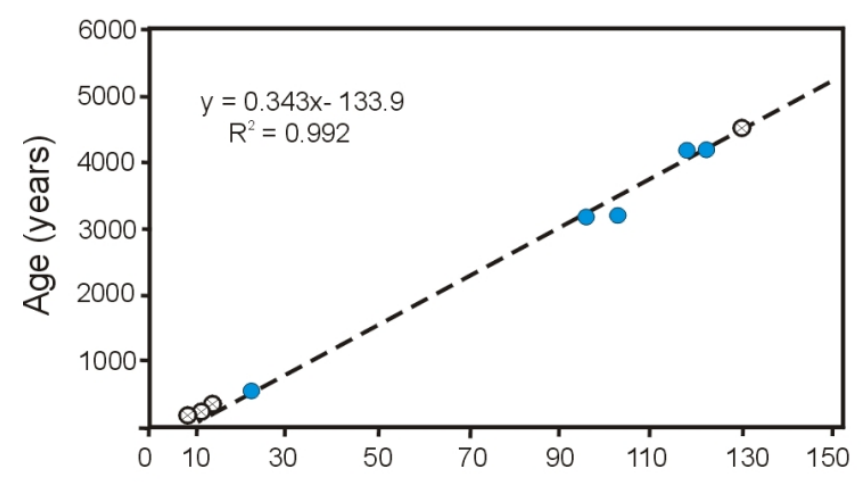

(b) Blue OSL Signal (photon counts $\times 10^{3}$ )

Figure 12 (a) A plot of luminescence signal versus depth in a pit excavated in an agricultural terrace in the Judean Highlands of Israel . Also shown are sampling positions of samples dated using standard OSL protocols. (b) A regression model of sample age versus blue OSL signal. The derived function was then used to calculate ages of samples of unknown age whose signals are indicated in Figure 12(a) (Adapted from Porat et al., 2019). 


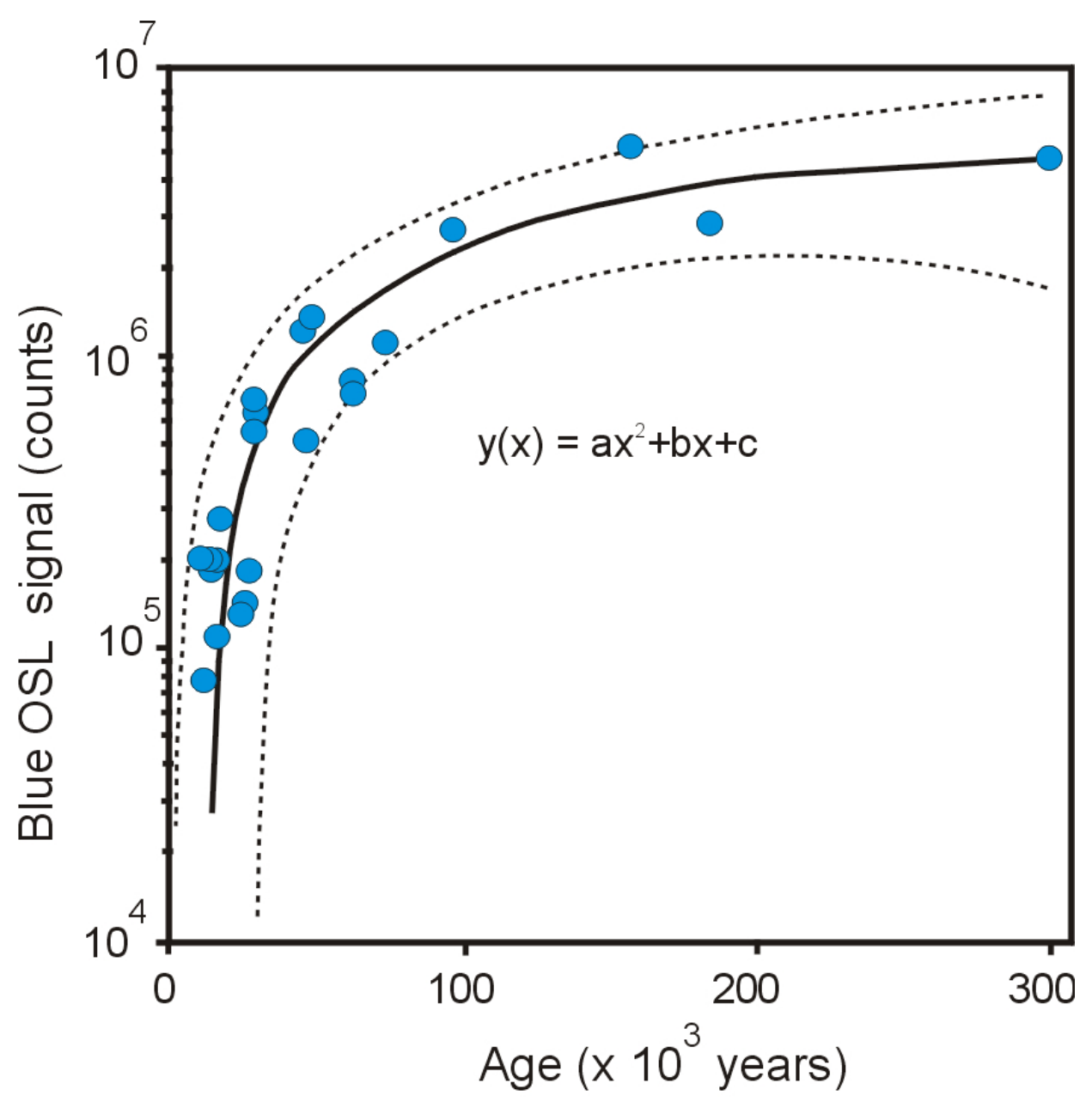

\section{- Sample of known age measured using portable OSL reader}

\section{- Quadratic curve fitted to data \\ :... 1-sigma uncertainty range}

Figure 13 Quadratic curve derived from portable OSL reader signals plotted against ages established using radiocarbon and standard OSL procedures. Data shown above are after sieving and removal of magnetic minerals using hand magnet (Adapted from Gray et al., 2018). 